ARTICLE

https://doi.org/10.1038/s41467-019-10131-1

\title{
TFIIE orchestrates the recruitment of the TFIIH kinase module at promoter before release during transcription
}

\author{
Emmanuel Compe ${ }^{1}$, Carlos M. Genes ${ }^{1}$, Cathy Braun ${ }^{1}$, Frederic Coin ${ }^{1} \&$ Jean-Marc Egly ${ }^{1}$
}

In eukaryotes, the general transcription factors TFIIE and TFIIH assemble at the transcription start site with RNA Polymerase II. However, the mechanism by which these transcription factors incorporate the preinitiation complex and coordinate their action during RNA polymerase II transcription remains elusive. Here we show that the TFIIE $\alpha$ and TFIIE $\beta$ subunits anchor the TFIIH kinase module (CAK) within the preinitiation complex. In addition, we show that while RNA polymerase II phosphorylation and DNA opening occur, CAK and TFIIE $\alpha$ are released from the promoter. This dissociation is impeded by either ATP- $\gamma \mathrm{S}$ or CDK7 inhibitor THZ1, but still occurs when XPB activity is abrogated. Finally, we show that the Core-TFIIH and TFIIE $\beta$ are subsequently removed, while elongation factors such as DSIF are recruited. Remarkably, these early transcriptional events are affected by TFIIE and TFIIH mutations associated with the developmental disorder, trichothiodystrophy.

\footnotetext{
${ }^{1}$ Department of Functional Genomics and Cancer, IGBMC, CNRS/INSERM/University of Strasbourg, BP 163, 67404 IIIkirch Cedex, C. U. Strasbourg, France. Correspondence and requests for materials should be addressed to E.C. (email: compe@igbmc.fr) or to J.-M.E. (email: egly@igbmc.fr)
} 
$\mathrm{n}$ eukaryotes, accurate transcription of protein-coding genes requires the synchronized action of multiple molecular actors. Among them, the general transcription factors (GTFs), including TFIIA, TFIIB, TFIID, TFIIE, TFIIF and TFIIH assemble at the transcription start site with RNA Polymerase II (Pol II) to form the preinitiation complex (PIC). While the different GTFs have been characterized almost 30 years ago, little is known about the chronological order of the enzymatic activities that occurr during transcription initiation. Nonetheless, a conventional assembly order for a Pol II-PIC emerged, in which TBP (TATA Binding Protein), as part of TFIID, initially binds to the promoter and is further stabilized by TFIIB and TFIIA. Pol II and TFIIF then align to make a stable TFIID-TFIIA-TFIIB-Pol II-TFIIF promoter complex, which is finally completed by the addition of TFIIE and TFIIH ${ }^{1,2}$.

The heterodimer TFIIE (composed of the TFIIE $\alpha$ and TFIIE $\beta$ subunits) seems to play a pivotal role in transcription by directly influencing the transition from initiation to elongation ${ }^{3,4}$. TFIIE interacts with different factors within the PIC, including Pol II ${ }^{5,6}$ as well as with DNA immediately upstream of the transcription bubble region ${ }^{7,8}$. Furthermore, TFIIE seems to influence TFIIH activity $^{9}$, although it is not clear how this molecular process can occur.

TFIIH, which also intervenes during the nucleotide excision repair (NER) pathway, contains 10 subunits that can be resolved into two sub-complexes: the Core (composed of the subunits $\mathrm{XPB}, \mathrm{p} 62, \mathrm{p} 52, \mathrm{p} 44, \mathrm{p} 34$, and TTDA) and the CAK (containing the CDK7 kinase, MAT1, and Cyclin H). Both sub-complexes interact with XPD subunit, whose ATPase/helicase activity promotes DNA opening during NER ${ }^{10,11}$. The role of TFIIH during transcription initiation mainly relies on its XPB and CDK7 enzymatic activities, XPD having essentially a structural function $^{12}$. Observations revealed that XPB is not a conventional helicase ${ }^{13,14}$, but an ATPase/translocase that pumps downstream DNA toward the Pol II to generate torsional and mechanical strain, leading to the formation of the DNA bubble ${ }^{15-18}$. For its part, CDK7 phosphorylates the serine 5 (Ser5-P) of the Cterminal domain (CTD) of the largest Pol II subunit (RPB1), thus contributing to promoter escape ${ }^{19-21}$. The CDK7 action is not restricted to Pol II, since other transcription factors are targeted by this kinase, including nuclear receptors (NRs), such as the retinoic acid receptor alpha $(\mathrm{RAR} \alpha)^{22}$

A better understanding of the transcription mechanism arose from the study of diseases related to mutations in genes encoding transcription machinery components (e.g. TFIIH, Mediator, etc. $)^{23,24}$. Remarkably, mutations within the TFIIE $\beta$ subunit recently have recently been associated with trichothiodystrophy (TTD), an autosomal recessive developmental disorder mainly related to TFIIH mutations (mostly in ERCC2/XPD gene and few cases in ERCC3/ $X P B$ or GFT2H5/p8/TTD- $A$ genes) and characterized by brittle hairs with alternating dark and light ("Tiger tail") banding with polarized microscopy and low content of sulfur-containing amino acids 25,26 . TFIIE/TTD patients, like TFIIH/TTD patients also have dry, ichthyotic skin, short stature, microcephaly, cerebellar dysfunction, developmental delay, happy engaging personality, attention deficit hyperactivity disorder, and myopia ${ }^{27}$.

Our work reveals an unexpected dynamic process during which TFIIE $\alpha$ and TFIIE $\beta$ act as key factors to recruit the CAK of TFIIH within the PIC. Furthermore, we show that Pol II phosphorylation is accompanied by the release of the CAK and TFIIEa from the promoter, a process that takes place before DNA opening. Once the CAK and TFIIEa are released, RNA synthesis is initiated, a process during which the Core-TFIIH and TFIIE $\beta$ are also removed while elongation factors including DRB sensitivity-inducing factor (DSIF) are recruited. Strikingly, TTDrelated mutations in either XPD or the TFIIE $\beta$-coding gene
(GTF2E2) similarly disrupt these processes, which could explain why alterations of TFIIE or TFIIH lead to the same clinical syndrome.

\section{Results}

Defective TFIIE alters PIC formation. Knowing that TTD cells with mutations in either XPB, XPD, or p8/TTD-A exhibit a decrease in the cellular concentration of $\mathrm{TFIIH}^{28,29}$, we first examined the levels of components of the transcription machinery in fibroblasts isolated from TTD patients bearing either TFIIE $\beta / A 150 P$ or /D187Y mutations. Western Blot analysis showed that both $\alpha$ and $\beta$ subunits of TFIIE were reduced in TTD/TFIIE-mutated cells when compared to wild-type fibroblasts (Fig. 1a). Such deficiency was only observed for TFIIE, since no reduction of the cellular concentration of TFIIH (visualized by XPB, XPD, and CDK7) or for the other GTFs, namely TFIIA (p35), TFIIB (p33), TFIID (TAF1, TAF4, and TBP), TFIIF (RAP74 and RAP30), and Pol II (visualized by the hypo-IIA and hyper-IIO phosphorylated form of its RPB1 subunit) was observed. We also evaluated the levels of TFIIE in TTD fibroblasts bearing the most common XPD mutations (Supplementary Fig. 1a). Surprisingly, we repeatedly observed a drop in TFIIE concentration in XPD/R722W fibroblasts and to a lower extend in XPD/R112H, which accompanied the decrease of TFIIH (Supplementary Fig. 1b).

We next investigated whether the expression of protein-coding genes was affected by TFIIE mutations, using the all-trans retinoic acid (t-RA) inducible $R A R \beta 2$ gene as a model. After t-RA treatment, the pattern of RAR $\beta 2$ mRNA synthesis was reduced in both IIE $\beta / A 150 P$ and IIE $/$ D $187 Y$ fibroblasts when compared with wild-type cells (Fig. 1b, c). Interestingly, similar profiles of RAR $\beta 2$ deregulation were observed in XPD/R112H and /R722W fibroblasts (Supplementary Fig. 1c, d), revealing recurrent transcriptional failures in TTD.

Chromatin immunoprecipitation (ChIP) assays were then performed to determine whether deficiencies occurred during the PIC assembly. In wild-type fibroblasts, the recruitment of both Pol II (visualized by its RPB1 subunit, Fig. 1d, e) and TFIIH (CDK7, Fig. 1f, g) at the RAR $\beta 2$ promoter highly increased at $6 \mathrm{~h}$, then decreased $8 \mathrm{~h}$ post-treatment, which perfectly paralleled the profile of the RAR $\beta 2$ mRNA synthesis (panels $b$ and $c$ ). Conversely, in TFIIE-deficient fibroblasts, Pol II and TFIIH were barely recruited $6 \mathrm{~h}$ post-treatment, despite their unchanged cellular concentrations (Fig. 1a). Contrary to what was observed in normal fibroblasts, the Pol II and TFIIH recruitment tended to increase at $8 \mathrm{~h}$ in TFIIE-deficient fibroblasts. Defective recruitment of Pol II and TFIIH also occurred in XPD/R112H and XPD/ R722W cells (Supplementary Fig. 1e, f, g, h). Surprisingly, we repeatedly observed that TFIIE, already detected at $t=0$ on the RAR $\beta 2$ promoter, progressively decreased in normal fibroblasts (Fig. 1h, i, Supplementary Fig. 1i, j); we however noticed a recurrent lower recruitment of TFIIE in TTD/TFIIE as well as in TTD/XPD fibroblasts.

In order to investigate whether TFIIE mutation might itself affect the expression of protein coding genes, homozygous knockin KI-IIE $\beta / A 150 \mathrm{P}$ cells were generated from the U2OS cell line by using CRISPR/Cas9 methodology (Supplementary Fig. 2a; see "Methods"); non-mutated U2OS cells (IIE $\beta /$ WT) were used as control. We first observed that the insertion of the missense mutation c.448G >C [p.Ala150Pro] within the GTF2E2 gene that encodes TFIIE $\beta$ was sufficient to drastically reduce the cellular levels of both $\alpha$ and $\beta$ subunits of TFIIE complex (Supplementary Fig. 2b). After t-RA treatment, we then observed that the pattern of RAR $\beta 2$ mRNA synthesis was reduced in KI-IIE $\beta / A 150 \mathrm{P}$ cells when compared with that observed in wild-type cells (Fig. 1j). 
a

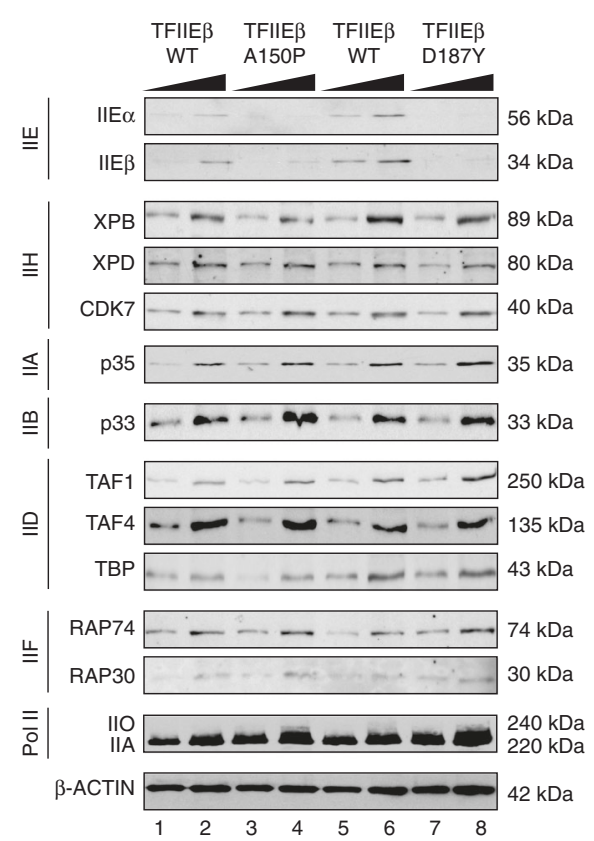

$R A R \beta 2$ gene expression

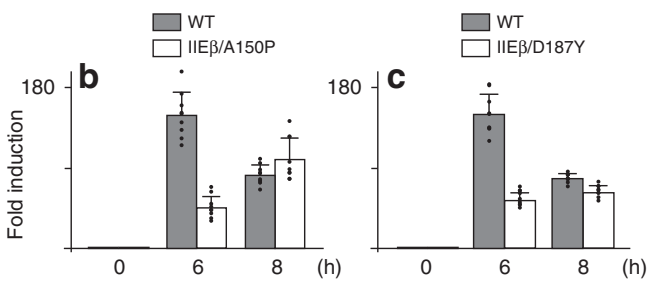

ChIP

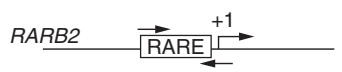

Pol II
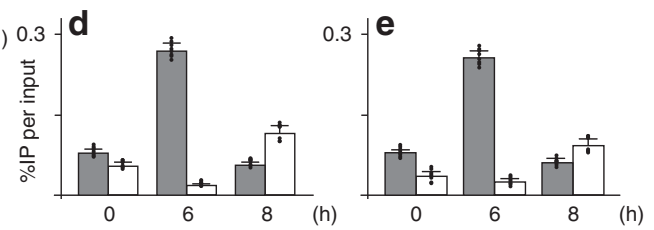

TFIIH

(CDK7)
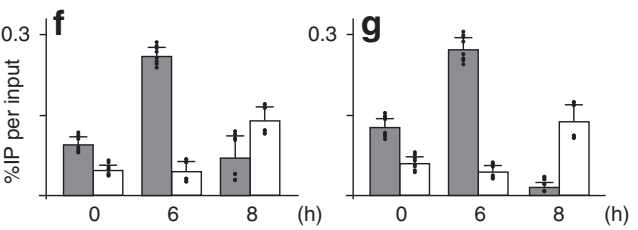

TFIIE
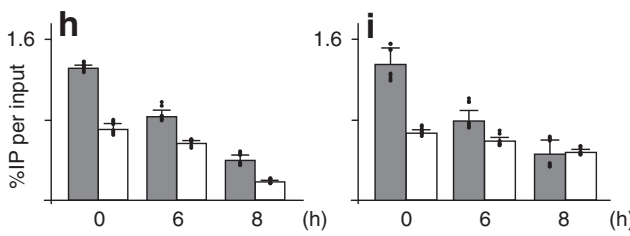
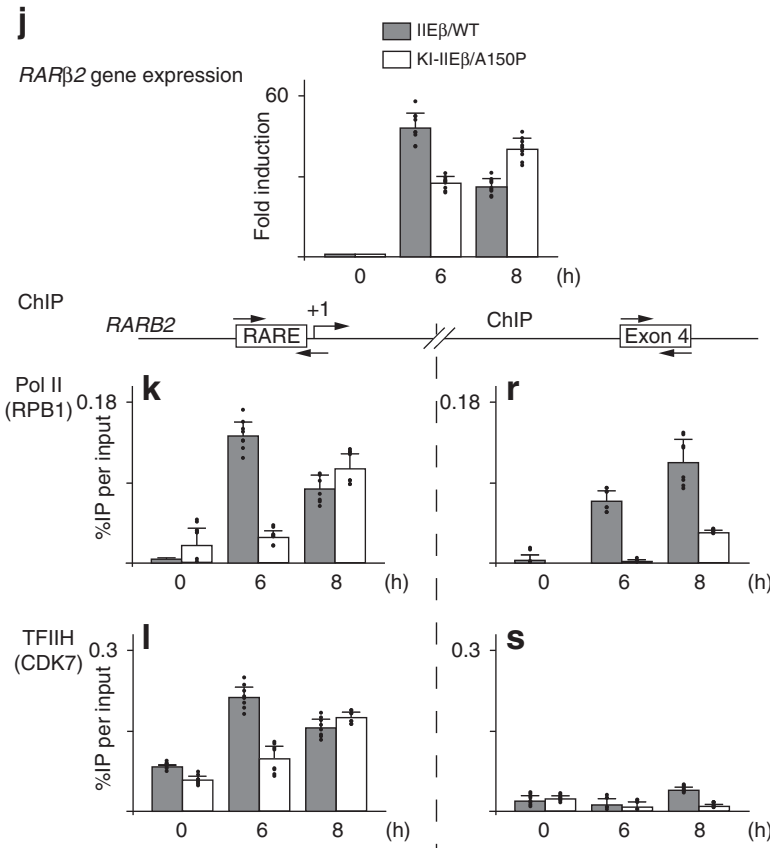

TFIIB
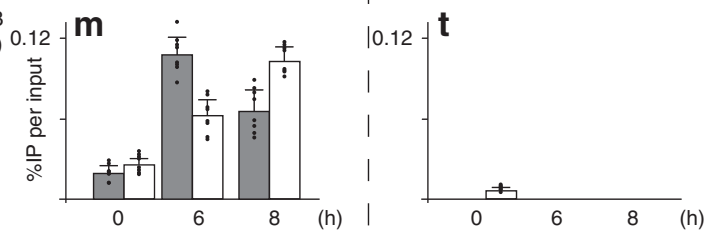

TFIIE
(IIE $\beta$ )

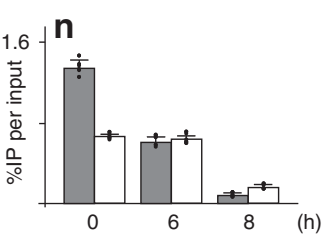

Ser5-P
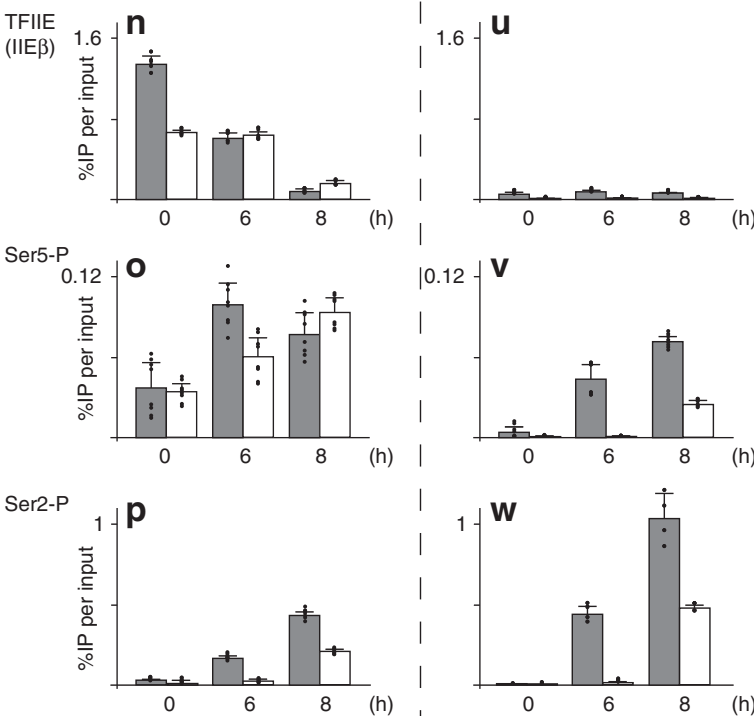

DSIF $0.3-$
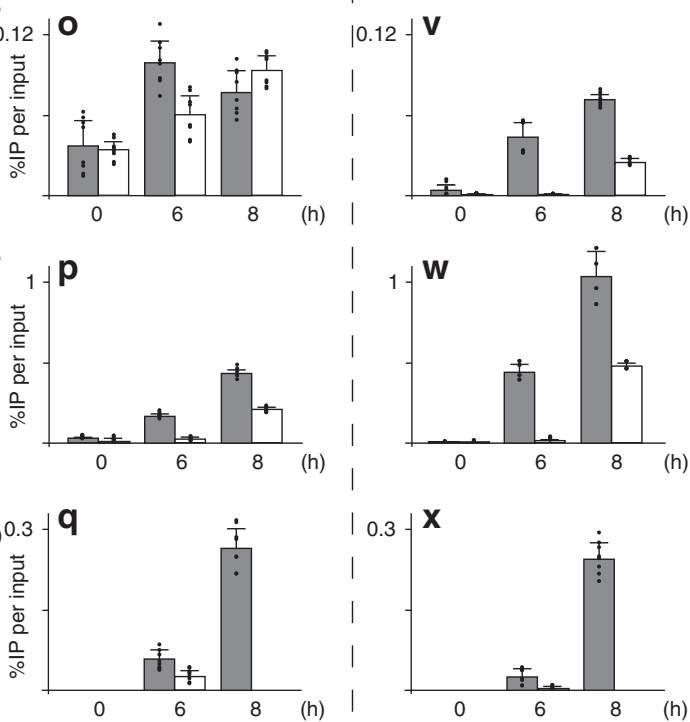

ChIP assays next revealed that the recruitment of Pol II (visualized by the presence of RPB1), TFIIH (CDK7), and TFIIB (p33) was reduced in KI-IIE $\beta / A 150 \mathrm{P}$ cells when compared with that observed in IIE $\beta / W T$ cells (Fig. 1k, l, m), which correlated with the profile of RAR $\beta 2$ mRNA synthesis (Fig. 1j). Interestingly, and as previously observed (Fig. 1h), TFIIE (using antibodies raised against TFIE $\beta$ ) detected at $t=0$ was progressively

removed from RAR $\beta 2$ promoter in IIE $\beta /$ WT cells (Fig. 1n); its recruitment was however deficient in KI-IIE $\beta / A 150 \mathrm{P}$ cells. Furthermore, an improper Ser5-P of Pol II was observed at the RAR $\beta 2$ promoter (Fig. 1o). Interestingly, in IIE $\beta / \mathrm{WT}$ and KIIIE $\beta / A 150 \mathrm{P}$ cells, the nuclear receptor RARa was similarly recruited to its response element (RARE) after $6 \mathrm{~h}$ of t-RA treatment (Supplementary Fig. 2c); however, RARa remained at 
Fig. 1 Defective transcription in cells harboring TFIIE mutations. a Increasing amounts of whole-cell lysates isolated from fibroblasts of patients with TFIIE $\beta$ / A150P, /D187Y, and of unaffected parents were used for immunoblot analysis of TFIIE (IIE $\alpha$ and IIE $\beta$ ), TFIIH (XPB, XPD, and CDK7), TFIIA (p35), TFIIB (p33), TFIID (TAF1, TAF4, and TBP), TFIIF (RAP74 and RAP30), and Pol II (the hypo IIA- phosphorylated and hyper IIO-phosphorylated forms of RPB1). $\beta$-actin was used as loading control. b, c Wild-type (gray boxes) and TTD fibroblasts (open boxes) with the mutation IIE $\beta / A 150 P$ (panel b) and IIE $\beta / D 187 Y$ (panel c) have been treated with t-RA $(10 \mu \mathrm{M})$. Relative RARB2 gene expression have been measured by RT-PCR after 0,6 , and $8 \mathrm{~h}$ of t-RA treatment (panels $\mathbf{b}$ and $\mathbf{c}$ ). The mRNA levels were normalized to the $18 \mathrm{~S}$ RNA amount. The results $(n=9$, means \pm s.d.) are presented as $n$-fold induction relative to non-treated cells. $\mathbf{d}$-i: ChIP experiments have been done 0,6 , and $8 \mathrm{~h}$ post-t-RA treatment from wild-type (gray boxes) and TTD fibroblasts (open boxes) with the mutation IIE $\beta / A 150 P$ (panels $\mathbf{d}$, $\mathbf{f}$, and $\mathbf{h}$ ) and IIE $\beta / D 187 Y$ (panels $\mathbf{e}, \mathbf{g}$, and $\mathbf{i}$ ) to analyze the recruitment of Pol II (RPB1, $\mathbf{d}$ and $\mathbf{e}$ ), TFIIH (CDK7, $\mathbf{f}$ and $\mathbf{g}$ ) and TFIIE (TFIIE $\beta, \mathbf{h}$ and $\mathbf{i}$ ) at the RARB2 proximal promoter. The values ( $n=8$, means \pm s.d.) are expressed as percentage of immunoprecipitated DNA relative to the input. $\mathbf{j}$ Relative RAR $\beta 2$ gene expression after 0,6 , and $8 \mathrm{~h}$ of $\mathrm{t}$-RA treatment in IIE $\beta / \mathrm{WT}$ (gray boxes) and KI-IIE $\beta / \mathrm{A} 150 \mathrm{P}$ (open boxes) cells. The mRNA levels were normalized to the $18 \mathrm{~S}$ RNA amount. The results $(n=9$, means \pm s.d.) are presented as $n$-fold induction relative to non-treated cells. k-x ChIP experiments have been done 0,6 , and $8 \mathrm{~h}$ post-t-RA treatment from IIE $\beta / \mathrm{WT}$ (gray boxes) and KI-IIE $\beta / \mathrm{A} 150 \mathrm{P}$ (open boxes) cells. We analyzed at the RARB2 proximal promoter (panels $\mathbf{k}-\mathbf{q}$ ) and exon 4 (panels $\mathbf{r}-\mathbf{x}$ ) the recruitment of Pol II (RPB1, $\mathbf{k}, \mathbf{r})$, TFIIH (CDK7, I, s), TFIIB (p33, $\mathbf{m}, \mathbf{t}$ ), $\operatorname{TFIIE} \beta(\mathbf{n}, \mathbf{u}), \operatorname{Ser} 5-\mathrm{P}(\mathbf{o}, \mathbf{v}), \operatorname{Ser} 2-\mathrm{P}(\mathbf{p}, \mathbf{w})$, and DSIF (SPT5, $\mathbf{q}, \mathbf{x})$. The values $(n=8$, means \pm s.d.) are expressed as percentage of immunoprecipitated (IP) DNA relative to the input. Source data are provided as a Source Data file

the promoter $8 \mathrm{~h}$ post-treatment in KI-IIE $\beta / \mathrm{A} 150 \mathrm{P}$ cells, a situation not observed in normal cells. Moreover, the phosphorylation by CDK7 of the nuclear receptor RARa (which is a prerequisite for accurate expression of its corresponding target genes) ${ }^{22}$ was not modified in t-RA-treated KI-IEß/A150P cells (Supplementary Fig. 2d). These results suggest that the disruption of the RAR $\beta 2$ mRNA synthesis did not result from deficiency of the RARa-activation process, but rather from defects during PIC assembly.

We also investigated the possible consequences of a defective PIC formation for ongoing transcription. ChIP assays at exon 4 showed increased phosphorylation of elongating Pol II at Ser2 (Ser2-P) in IIE $\beta /$ WT cells after $6 \mathrm{~h}$ t-RA treatment (Fig. 1w). Persistence of phosphorylated elongating Pol II at $8 \mathrm{~h}$, when RAR $\beta 2$ mRNA tends to decrease, might be due to the cyclic profile of the $R A R \beta 2$ gene expression ${ }^{30}$ and the very distal position of exon 4 from the transcription start site $(\sim 140 \mathrm{~kb})$. Strikingly, Pol II phosphorylation was strongly disrupted in KIIIE $\beta / A 150$ P cells (Fig. 1w). It is worthwhile to notice that under our experimental conditions, neither TFIIB nor TFIIE (TFIIE $\beta$ ) were detected at exon 4 in normal and mutated cells (Fig. 1t, u); only background levels were detected for CDK7 (Fig. 1s). Finally, the recruitment of the transcription elongation factor DSIF ${ }^{31}$ (visualized via its SPT5 subunit) was clearly impaired in mutated cells (Fig. 1x), suggesting that the previously observed alterations in PIC formation influence transcriptional elongation. All together our data show how the arrival of the transcriptional machinery with Pol II and the release of TFIIE occur at the promoter of activated gene to engage RNA synthesis, and how TFIIE and TFIIH mutations notably disturb such coordinated events.

Mutations within TFIIE $\beta$ disrupt transcription initiation. We next asked how the TFIIE/TFIIH partnership occurred. Recombinant TFIIE (rIIE) containing rIIEa/WT subunit with either $\mathrm{rIIE} / \mathrm{WT}$, /A150P, or /D187Y were produced and purified (Fig. 2a). We first observed that addition of increasing amounts of either rIIE $\alpha \beta / \mathrm{WT}$, rIIE $\alpha \beta / \mathrm{A} 150 \mathrm{P}$, or $\mathrm{rIIE} \alpha \beta / \mathrm{D} 187 \mathrm{Y}$ in NER reaction (containing XPC, TFIIH, XPA, RPA, XPF/ERCC1, and XPG as well as a cisplatinated substrate $)^{32}$, did not influence the removal of the damaged oligonucleotides (Supplementary Fig. 3a). On the contrary, purified recombinants rIIH-XPD/ R112H or rIIH-XPD/R722W (Fig. 2b) drastically prevented NER (Supplementary Fig. 3b). This strongly suggested that TFIIE did not modulate in vitro NER activity.

We next investigated the consequences of TFIIE mutations during in vitro transcription assays. The rIIEs were incubated together with the adenovirus major late promoter (AdMLP, run- off of 309nt), TFIIA, TFIIB, TFIID (TBP), TFIIF, and TFIIH as well as Pol II ${ }^{12}$. We observed that 5, 10, and 20 min after addition of nucleoside triphosphates (NTPs, including radiolabelled CTP), rIIEa $3 / A 150 \mathrm{P}$ and /D187Y led to a lower RNA synthesis than rIIEa $\beta /$ WT (Fig. 2c). The defect was slightly more pronounced with rIIEaß/A150P than /D187Y (compare lanes 5-7 to 8-10). Similarly, transcriptional defect was observed in the presence of rIIH-XPD/R112H or /R722W (Fig. 2d, lanes 5-7 and 8-10, respectively).

To evaluate whether TFIIE mutations might interfere with the first steps of transcription, we performed in vitro abortive initiation assays (Fig. 2e), during which we measured trinucleotide synthesis initiated by a reporter priming dinucleotide RNA (CpA) hybridized to the transcription start site of the AdMLP 33,34 . Upon addition of radiolabelled CTP and increasing amounts of either rIIEaß/A150P or /D187Y, trinucleotide (CpApC) synthesis was impaired (lanes 5-7 and 8-10). The defect was greater with rIIEaß/A150P than /D187Y, which paralleled what was observed during in vitro run-off assays (Fig. 2c). Interestingly, the difference between the two mutated forms of TFIIE was more pronounced in run-off than in abortive assays, suggesting that early transcriptional steps might be differently affected by rIIEa $\beta / A 150 P$ and $\mathrm{rIIE \alpha} / \mathrm{D} 187 \mathrm{Y}$; the latter ensures initiation but may fail at subsequent steps. Finally, impairment of trinucleotide synthesis was also observed when increasing amounts of $\mathrm{rIIH}-\mathrm{XPD} / \mathrm{R} 112 \mathrm{H}$ or $/ \mathrm{R} 722 \mathrm{~W}$ together with rIIEaß/WT were used (Fig. 2f).

Together, the above results underlined the essential role of TFIIE during transcription initiation. In addition, it seems that TTD-related mutations within TFIIE and TFIIH both disrupt the early steps of transcription initiation.

TFIIEa is essential to incorporate the CAK within the PIC. We then investigated the role of TFIIE during PIC formation. Biotinylated AdMLP was immobilized to streptavidin beads and incubated with all the GTFs (TFIIA, TFIIB, TBP, TFIIF, TFIIH), Pol II, and either rIIE $\alpha \beta / W T$, rIIE $\alpha \beta / A 150 P$, or rIIE $\alpha \beta / D 187 Y$ (Fig. 3a). After several washes, western blot analysis of the remaining proteins bound to DNA showed that Pol II (revealed by its RPB2 subunit), TFIIF (RAP74), and TBP were associated with DNA in the absence of TFIIE (lane 2); the signals corresponding to the Core-TFIIH (XPB) and the XPD bridging component of TFIIH were however reduced, with an even more severe for CAK (CDK7). Interestingly, addition of rIIE $\alpha \beta / \mathrm{WT}$ promoted the recruitment of TFIIH (lane 3), consistent with the observation that TFIIH co-immunoprecipitated with rIIE $\alpha \beta / \mathrm{WT}$ (Fig. 3b, lanes 1 and 2). Strikingly, rIIEaß/A150P and /D187Y reduced the binding of CAK (CDK7) (Fig. 3a, compare lanes 4 

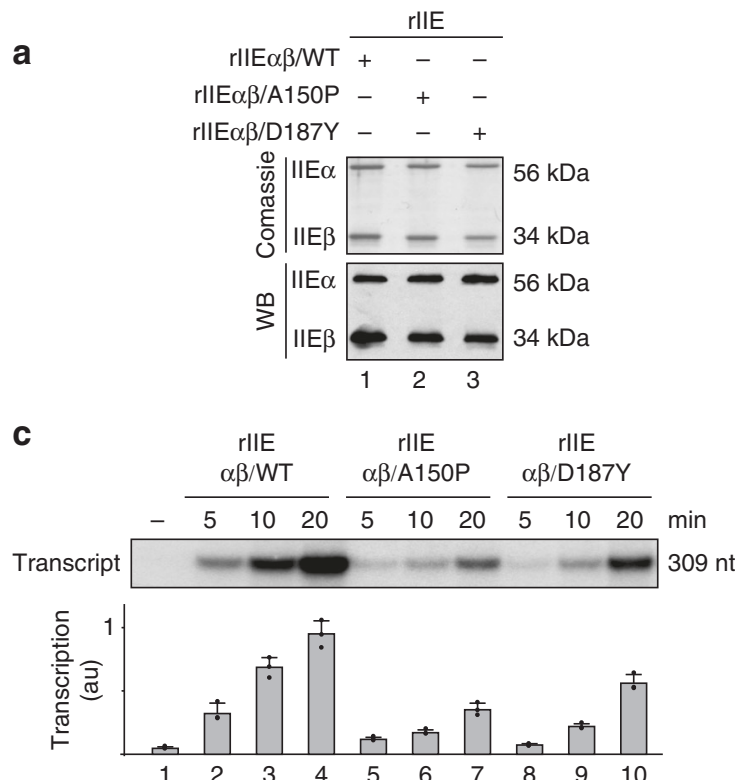

e
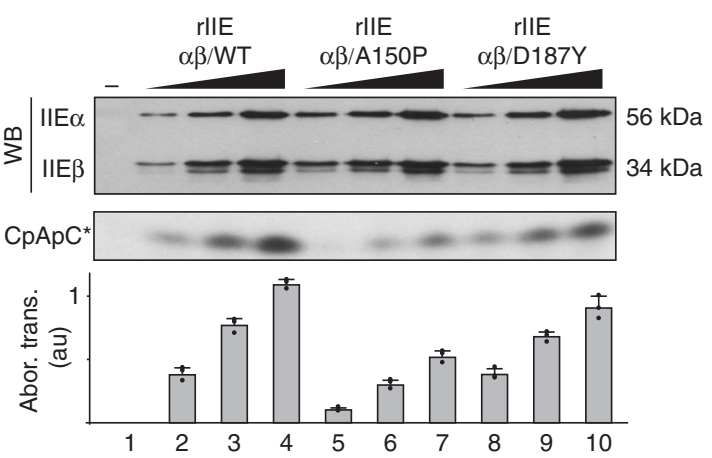

b

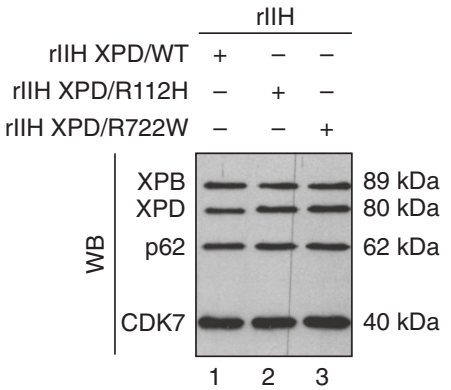

d
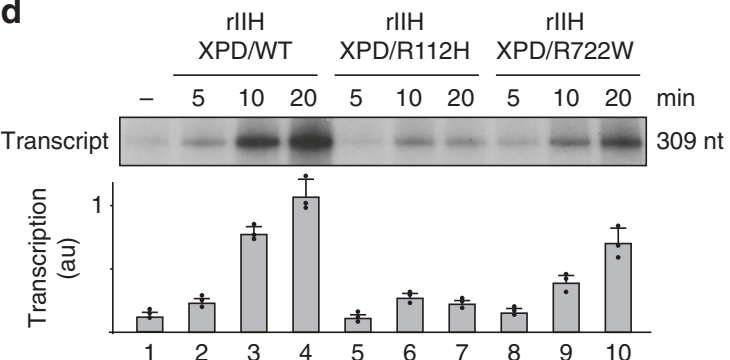

f
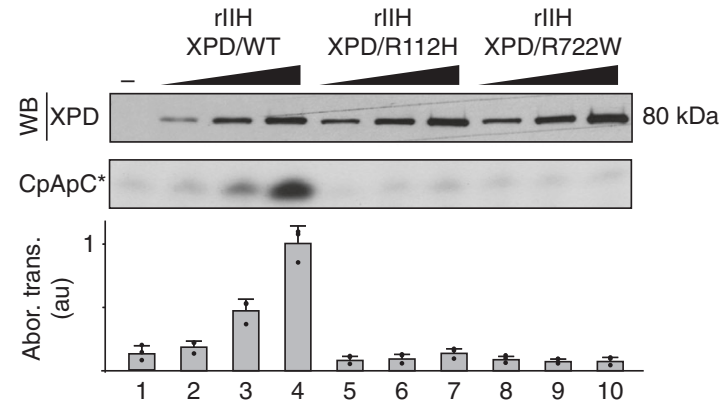

Fig. 2 Defective TFIIE and TFIIH affect transcription initiation. a Production of the recombinant mutated forms of TFIIE (rIIE). Equal amounts of purified rlIE were resolved by SDS-PAGE. Coomassie blue staining gel (top panel) and western blot (WB, bottom panel) with antibodies raised against TFIIE $\alpha$ and TFIIE $\beta$ are shown. $\mathbf{b}$ Production of the recombinant mutated forms of TFIIH (rIIH). Equal amounts of purified rllHs were resolved by SDS-PAGE and immunoblotted for XPB, XPD, p62, and CDK7. c, d Purified rllEs (c) and rllHs (d) were added to in vitro reconstituted transcription systems, as indicated. After addition of NTPs (including radiolabelled CTP), the transcription activity was assessed after 5, 10, and 20 min of incubation. The length of the corresponding transcript (309nt) is indicated on the right side. The signals were quantified ( $n=3$, means \pm s.d.) and plotted in arbitrary units (a.u.). The results are representative of three independent experiments. e, $\mathbf{f}$ Abortive transcription assays using rllEs (e) and rllHs (f). After preinitiation complex assembly using equivalent amounts of the different purified rllEs and rllHs (as revealed by western blots), phosphodiester bond synthesis was initiated by the addition of priming dinucleotides CpA and radiolabelled CTP. After 30 min of incubation, the radioactive trinucleotide (CpApC ${ }^{\star}$ ) synthesis was stopped, quantified $(n=3$, means \pm s.d.) and plotted in arbitrary units (a.u.). The results are representative of two independent experiments. Source data are provided as a Source Data file

and 5 with lane 3 and histogram), related to the lower capacity of the mutated rIIEs to entirely co-immunoprecipitate with TFIIH (Fig. 3b, lanes 3-6).

The above observations prompted us to explore the involvement of TFIIE subunits in the recruitment of TFIIH within the PIC. In the absence of $\mathrm{rIIE} \beta / \mathrm{WT}$, rIIE $\alpha / \mathrm{WT}$ was unable to bind the AdMLP/Pol II/TBP/TFIIA/TFIIB/TFIIF complex (Fig. 3c, lane 2). Strikingly, whereas TFIIH interacted with $\mathrm{rIIE} \beta / \mathrm{WT}$ rather than $\mathrm{rIIE} / \mathrm{WT}$ in solution (Fig. 3d, lanes 2 and 4 ), both TFIIE subunits were required to fully recruit entire TFIIH, including CAK, to the AdMLP (Fig. 3c, lane 7). These observations may be related to the ability of rIIEa $\beta / \mathrm{WT}$ to interact with TFIIH (Fig. 3b), in particular with the CAK (Supplementary Fig. 4) ${ }^{35}$. Coimmunoprecipitation between TFIIE and TFIIH components were slightly reduced in the presence of rIIE $\alpha \beta / A 150 \mathrm{P}$ or rII $\alpha \beta / \mathrm{D} 187 \mathrm{Y}$ variants (Fig. 3b, lanes 3-6). Interestingly, rIIE $\beta$ mutations weakened interaction with rIIEa/ WT, as observed at high salt concentration (Fig. 3e), possibly due to the position of the TFIIE $\beta$ mutations in the $\mathrm{WH} 2$ domain which interacts with TFIIEa ${ }^{36,37}$. For their part, XPD/TTD mutations affected the integrity of TFIIH by destabilizing the interaction between the CAK (CDK7) and the Core (XPB) subcomplexes (Fig. 3f). As a consequence, the recruitment of the CAK at the AdMLP was reduced, as observed for XPD/R722W (Fig. 3g, compare lanes 5 to 3 ).

Altogether, the above data underlined the role played by TFIIE $\beta$ to anchor TFIIEa, which further allows incorporation of the whole TFIIH within the PIC. Additionally, we showed that mutations in TFIIE and TFIIH disturb the integration of these factors within the PIC.

ATP promotes the release of TFIIEa and the CAK. We next investigated how TFIIE and TFIIH cooperate to phosphorylate Pol II and to open DNA during transcription ${ }^{13,38}$. Addition of ATP to the PIC formation assay (containing the immobilized 
a

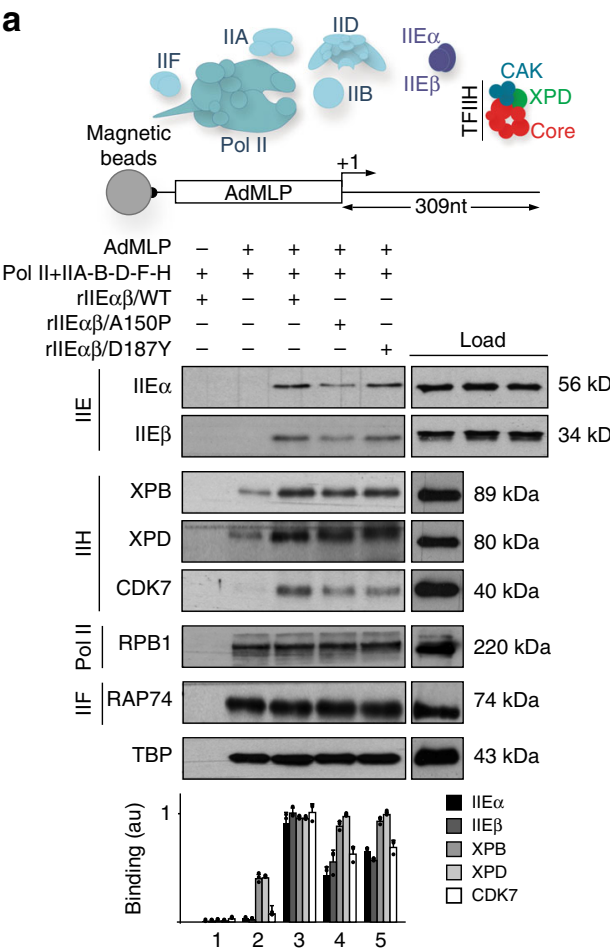

b

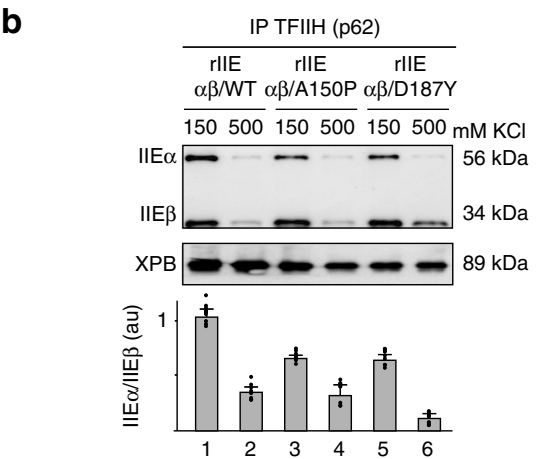

C

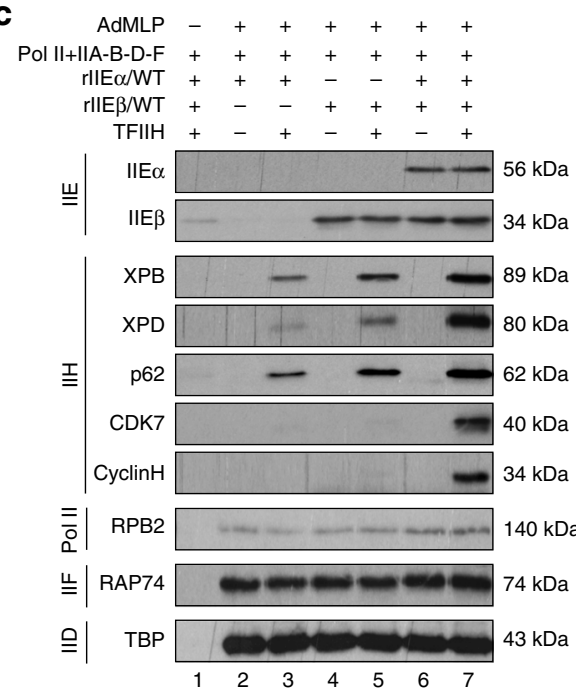

AdMLP, Pol II, and all the GTFs including rIIEa $\beta /$ WT) led to accumulation of Ser5-P of Pol II (Fig. 4a, lane 3). Furthermore, in such conditions, the CAK (visualized by CDK7 and Cyclin $\mathrm{H}$ ) and TFIIE $\alpha$ were separated from the Core-TFIIH and TFIIE $\beta$, which were maintained at the DNA template together with Pol II (RPB2) and TFIIF (RAP74). In presence of ATP- $\gamma$ S, whose d

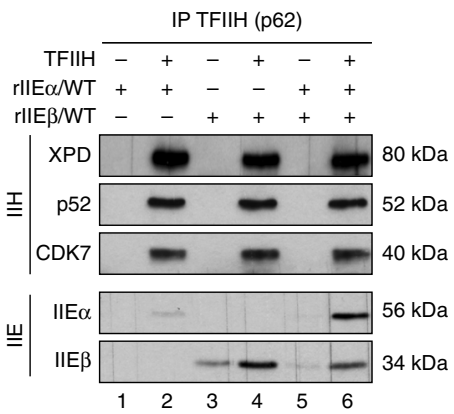

e

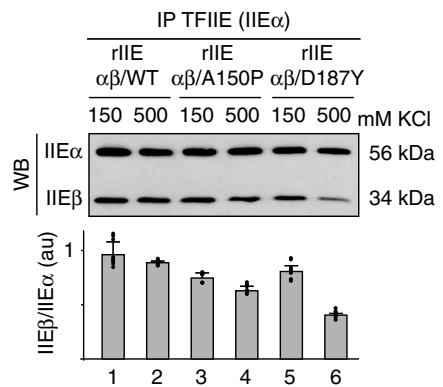

f

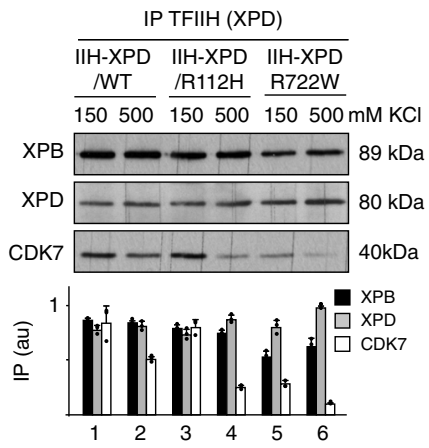

g $\begin{array}{rlllll}\text { AdLMP } & - & + & + & + & + \\ \text { Pol II+IIA-B-D-E-F } & + & + & + & + & + \\ \text { Core+CAK } & + & + & + & + & + \\ \text { XPD/WT } & + & - & + & - & - \\ \text { XPD/R112H } & - & - & - & + & - \\ \text { XPD/R722W } & - & - & - & - & +\end{array}$

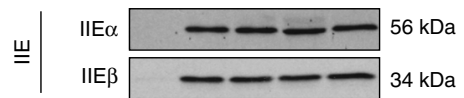

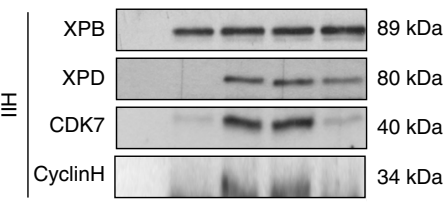

$\overline{\bar{O}} \mid \mathrm{RPB2} \longrightarrow 140 \mathrm{kDa}$

宸 RAP74 $74 \mathrm{kDa}$

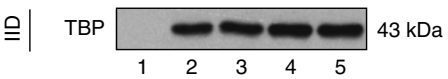

hydrolysis is very low compared to ATP, neither Pol II phosphorylation nor the release of the CAK and TFIIEa occurred (lane 4). Interestingly, Pol II phosphorylation and TFIIEa release were not observed in absence of the CAK (Fig. 4b, lane 3). Immobilized AdMLP template was next incubated with Pol II and all the GTFs including rIIEa $\beta / \mathrm{WT}$ and $\mathrm{rIIH} / \mathrm{WT}$ in presence of 
Fig. 3 Defective TFIIE and TFIIH alter the preinitiation complex. a Biotinylated AdMLP bound to streptavidin magnetic beads was incubated with Pol II, TFIIA, TFIIB, TFIID (TBP), TFIIF, and TFIIH, in the presence (+) of either rIIE $\alpha \beta / W T$, rllE $\alpha \beta / A 150 P$, or rlIE $\alpha \beta / D 187 Y$. After washes, the binding of different factors was evaluated by immunoblotting. The loading proteins used for PIC formation and the different rlIEs (in the following order rllE $\alpha \beta / W T$, rlIE $\alpha \beta /$ $\mathrm{A} 150 \mathrm{P}$, and $\mathrm{rlIE} \alpha \beta / \mathrm{D} 187 \mathrm{Y}$ ) are indicated at the right part of the panel (load). The signals for IIE $\alpha, I I E \beta, X P B, X P D$, and CDK7 were quantified ( $n=3$, means \pm s.d.) and plotted in arbitrary units (a.u.). The results are representative of three independent experiments. $\mathbf{b}$ Immunoprecipitated rllH/WT (using antip62) was incubated with rllEs at 150 and $500 \mathrm{mM} \mathrm{KCl}$. After washes, the coimmunoprecipitated proteins were resolved by SDS-PAGE and blotted with anti-XPB, anti-IIE $\alpha$, and anti-IIE $\beta$. Graph shows in arbitrary units (a.u.) the ratio IIE $\alpha / I I E \beta$ for each rlIE. The results are representative of three independent experiments. c Pol II, TFIIA, TFIIB, TFIID (TBP), and TFIIF were incubated with biotinylated AdMLP bound to streptavidin beads in presence ( + ) of rIIE $\alpha$ / WT, rllE $\beta / W T$, and TFIIH. Immunoblot analysis has been done with different antibodies, as indicated. d TFIIH was immunoprecipitated with anti-p62 and incubated $(+)$ with rlIE $\alpha /$ WT and/or rlIE $\beta / W T$. After washes, immunoblot analysis of TFIIH (XPD, p52, CDK7) and TFIIE (IIE $\alpha$ and IIE $\beta$ ) has been done. e Recombinant rlIE $\alpha / W T$, co-produced with either rIIE $\beta / W T$ (lanes 1 and 2), rlIE $\beta / A 150 P$ (lanes 3 and 4) or rllE $\beta / D 187 Y$ (lanes 5 and 6), was immunoprecipitated at 150 and $500 \mathrm{mM} \mathrm{KCl}$. After washes, the proteins were resolved by SDS-PAGE and blotted with antibodies against IIE $\alpha$ and IIE $\beta$. Graph shows the ratio $\| \mathrm{E} \beta / \mathrm{IIE} \alpha(n=3$, means \pm s.d.) in arbitrary units (a.u.). The results are representative of three independent experiments. $\mathbf{f} r \mathrm{rl} H$ containing either XPD/WT (lanes 1 and 2), XPD/R112H (lanes 3 and 4), or XPD/R722W (lanes 5 and 6) were immunoprecipitated with anti-XPD and incubated at 150 and $500 \mathrm{mM} \mathrm{KCl}$. After washes, the bound proteins were resolved by SDS-PAGE. The immunoprecipitated signals (IP) for XPB, XPD, and CDK7 were quantified ( $n=3$, means \pm s.d.) and plotted in arbitrary units (a.u.). The results are representative of three independent experiments.

g Streptavidin magnetic beads were incubated (when indicated, + ) with biotinylated AdMLP, Pol II, TFIIA, TFIIB, TFIID (TBP), rIIE $\alpha \beta /$ WT, TFIIF, the CoreTFIIH, the CAK and either XPD/WT, /R112H, or /R722W. After washes, immunoblot analysis of different factors has been done. The results $(n=3$, means \pm s.d.) are representative of three independent experiments. Source data are provided as a Source Data file

ATP and THZ1, a specific CDK7 inhibitor that covalently targets a cysteine residue located outside of the canonical kinase domain $^{39}$. In these conditions, Pol II remained unphosphorylated and both TFIIEa and the CAK were maintained at the DNA template (Fig. 4c, lanes 4 and 5). Taken together, the above data strongly suggested that once Pol II is phosphorylated by CDK7, TFIIE a well as the CAK are no longer required at the promoter.

We next examined whether promoter opening was required for Pol II phosphorylation and the release of TFIIEa and the CAK. In our in vitro assays, the addition of triptolide (TPL), a chemical compound known to inhibit the ATPase activity of XPB and to block transcription (Supplementary Fig. 5a) ${ }^{40}$, did not modify the ATP-dependent release of the CAK and TFIIEa (Fig. $4 \mathrm{~d}$ ). Furthermore, Ser5-P of Pol II was maintained (lane 5). Similarly, we also observed that the release of the CAK and TFIIEa still occurred in the presence of the mutated form rIIH-XPB/Fs740 (Fig. 4e, lanes 4 and 5), which prevented the XPB activity and affected transcription (Supplementary Fig. 5b) ${ }^{41}$. As a further control, we also found that $\mathrm{rIIH}-\mathrm{XPB} / \mathrm{K} 346 \mathrm{R}$, which harbors a mutation in the ATPase Walker motif that prevents ATP hydrolysis and disrupts transcription (Supplementary Fig. 5c) ${ }^{42,43}$, did not prevent the Pol II phosphorylation, as well as the release of the CAK and TFIIEa (Fig. 4f, lanes 4 and 5). Altogether, the above data suggested that promoter opening was not a prerequisite for Pol II phosphorylation nor the release of the CAK and TFIIEa.

The CAK is released during RNA synthesis. We next tested whether the PIC was able to promote RNA synthesis after having released the CAK and TFIIEa. After incubation of the immobilized AdMLP template with Pol II and all the GTFs, we added ATP as indicated (Fig. 5a). After washes, the remaining complex (containing the IIO-phosphorylated form of RPB1, lane 3) was incubated with all the NTPs to allow RNA synthesis (lane 4, 309nt run-off transcript). In such conditions, we observed a release of the CAK (as vizualized by CyclinH) and TFIIEa; the Core-TFIIH was partially released (as vizualized by XPB) while TFIIE $\beta$ was maintained at the promoter. It is noteworthy that less RNA synthesis occurred after an ATP pre-incubation was done (Fig. 5b), which was in agreement with previous observations showing that the activity of PICs was reduced by exposure to ATP alone ${ }^{44,45}$. Since TFIIE can reactivate Pol II after ATP preincubation $^{46}$, we supplemented with increasing amounts of IIEa following ATP treatment, washes, and addition of NTPs (Fig. 5c). This increased the presence of TFIIE $\alpha$ at the promoter (lanes 5 and 6), and RNA synthesis (Fig. 5d, lanes 4 and 5), as previously observed $^{46}$.

We next explored the fate of TFIIE $\beta$ during transcription. To determine whether additional factors might contribute to its release, the immobilized in vitro assay was supplemented with whole cell extracts (WCE, isolated from HeLa cells) in the presence of NTPs (Fig. 5e, f). While RNA synthesis occurred, we observed the complete removal of the Core-TFIIH (lane 3). Remarkably, the TFIIE $\beta$ subunit was also released, concomitant with the recruitment of the elongation factor DSIF (SPT5), as observed during ChIP experiments (Fig. 1q). TFIIE $\beta$ was partially removed in the presence of ATP alone (Fig. 5f, lane 3). However, the elongation factor DSIF (SPT5) was not recruited when compared to what obtained in presence of NTP (lane 4), suggesting that ATP addition was sufficient for TFIIEa eviction but that all four NTPs were needed for complete removal of TFIIE $\beta$ and DSIF recruitment.

Taken together, these results strongly suggested that TFIIEa and the CAK, followed by the Core-TFIIH and TFIIE $\beta$, are sequentially released from the promoter while elongation factors take place to pursue transcription.

TTD mutations prevent CAK recruitment. We next investigated how TTD mutations might perturb the transcription initiation process. Immobilized AdMLP was first incubated with Pol II, the GTFs (TFIIA, TFIIB, TBP, TFIIF, TFIIH) and either rIIE $\alpha \beta / \mathrm{WT}$, rIIEa $\beta / A 150 P$, or rIIE $\alpha \beta / D 187 Y$ in the presence of ATP (Fig. 6a). After washes, western blots showed that the ATP-dependent release of the CAK also occurred in presence of rIIEa $\beta / A 150 P$ and rIIEa/D187Y (lanes 4 and 6); it should be however noted that the CAK was recruited much less in the absence of ATP (lanes 3 and 5) as was previously observed (Fig. 3a). Similarly, the CAK was released upon ATP addition in the presence of TFIIH XPD/WT (Fig. 6b, lanes 2 and 3), XPD/R112H (lanes 4 and 5) and to some extent with XPD/R722W (lane 6 and 7). Consequently, TTD-causative mutations impaired the recruitment of the CAK subcomplex on transcription start sites.

In vitro transcription assays were also performed in the presence of the basal transcription machinery with the different rIIEs and rIIHs variants (Fig. 6c, d). Reactions were stopped 5, 10, and $20 \mathrm{~min}$ after addition of cold NTPs and analyzed by western blot using antibodies raised against Ser5-P of Pol II. The presence 
a

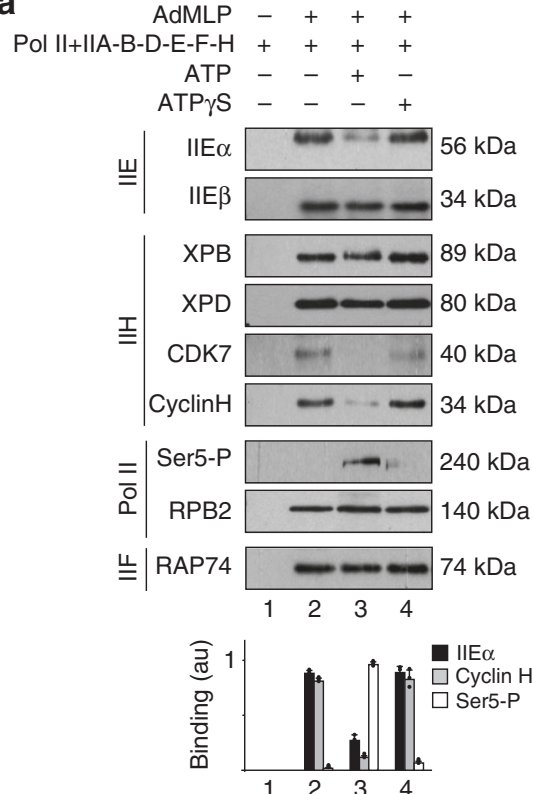

d

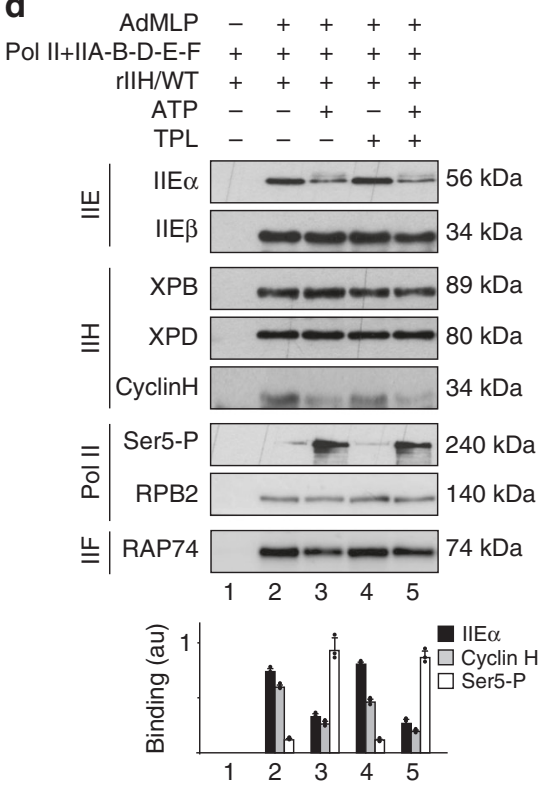

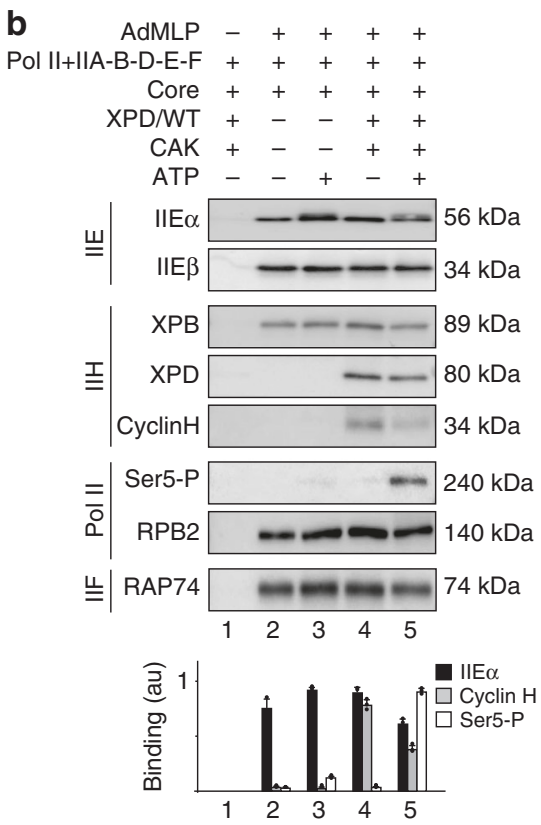

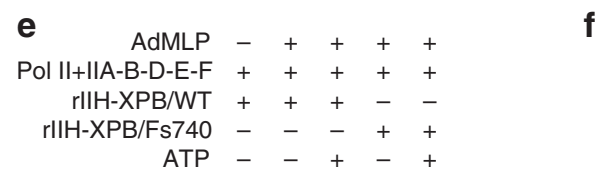

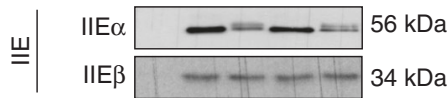

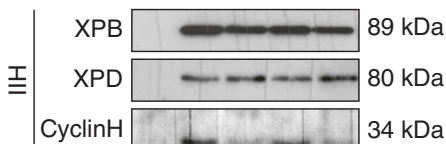

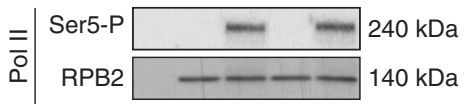

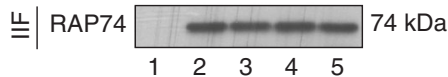

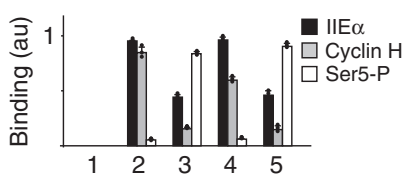

C

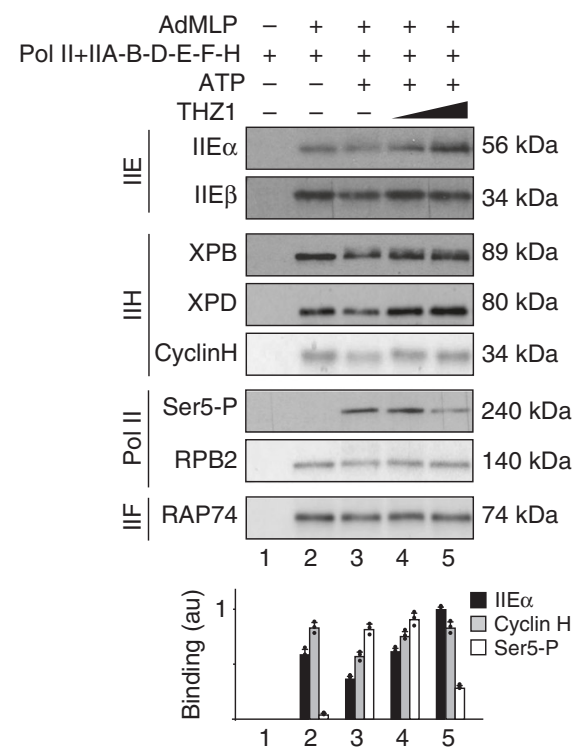

Pol

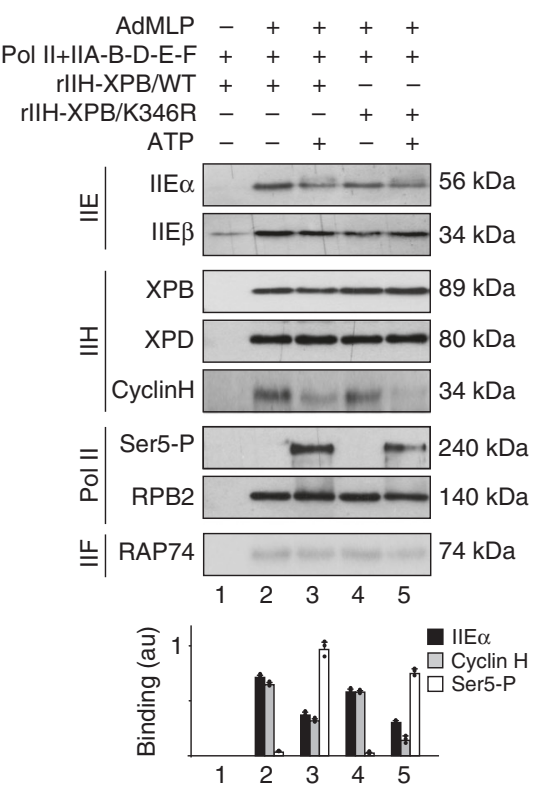

Fig. 4 TFIIE $\alpha$ and CAK releases and Pol II phosphorylation occur before DNA opening. a Streptavidin magnetic beads were incubated (+) with biotinylated AdMLP, Pol II, TFIIA, TFIIB, TFIID (TBP), rIIE $\alpha \beta /$ WT, TFIIF and rIIH/WT, in presence of either ATP (200 $\mu M)$ or ATP $\gamma S(200 \mu M)$. After washes, the binding of various factors has been evaluated by immunoblotting. The immunoblot signals for TFIIE $\alpha, \mathrm{CyclinH}$, and Ser5-P were quantified $(n=3$, means \pm s.d.) and plotted in arbitrary units (a.u.). The results are representative of three independent experiments. $\mathbf{b}$ Streptavidin magnetic beads were incubated (when indicated, + ) with biotinylated AdMLP, Pol II, TFIIA, TFIIB, TFIID (TBP), rIIE $\alpha \beta /$ WT, TFIIF, the Core-TFIIH, XPD/WT, and the CAK, in presence of ATP $(200 \mu \mathrm{M})$. After washes, immunoblot analysis was done for different proteins. The immunoblot signals for TFIIE $\alpha$, CyclinH, and Ser5-P were quantified $(n=3$, means \pm s.d.) and plotted in arbitrary units (a.u.). The results are representative of three independent experiments. c Streptavidin magnetic beads were incubated $(+)$ with biotinylated AdMLP, Pol II, TFIIA, TFIIB, TFIID (TBP), rIIE $\alpha \beta /$ WT, TFIIF, and rIIH/WT, in presence of ATP (200 $\mu M)$ and THZ1 $(1$ and $10 \mu \mathrm{M}$ ). The immunoblot signals for TFIIE $\alpha, \mathrm{CyclinH}$, and Ser5-P were quantified ( $n=3$, means \pm s.d.) and plotted in arbitrary units (a.u.). The results are representative of three independent experiments. $\mathbf{d}$ When indicated $(+)$, biotinylated AdMLP bound to streptavidin magnetic beads were preincubated $20 \mathrm{~min}\left(\right.$ at $25^{\circ} \mathrm{C}$ ) with the general transcription machinery (Pol II, TFIIA, TFIIB, TFIID (TBP), rIIE $\alpha \beta / \mathrm{WT}$, TFIIF, and rllH/WT) in presence of Triptolide (TPL, $10 \mu \mathrm{M})$ before addition of ATP $(200 \mu \mathrm{M})$. The immunoblot signals for TFIIE $\alpha, \mathrm{CyclinH}$, and Ser5-P were quantified $(n=3$, means \pm s.d. $)$ and plotted in arbitrary units (a.u.). The results are representative of three independent experiments. e Streptavidin magnetic beads were incubated ( + ) with biotinylated AdMLP, Pol II, TFIIA, TFIIB, TFIID (TBP), rIIE $\alpha \beta /$ WT, and TFIIF, in presence of ATP $(200 \mu M)$ and either rlIH-XPB/WT or /Fs740. The immunoblot signals for TFIIE $\alpha, C y c l i n H$, and Ser5-P were quantified $(n=3$, means \pm s.d.) and plotted in arbitrary units (a.u.). The results are representative of three independent experiments. f Streptavidin magnetic beads were incubated (+) with biotinylated AdMLP, Pol II, TFIIA, TFIIB, TFIID (TBP), rIIE $\alpha \beta /$ WT, and TFIIF, in presence of ATP $(200 \mu \mathrm{M})$ and either rIIH-XPB/WT or $/$ K346R. The immunoblot signals for TFIIE $\alpha$, CyclinH, and Ser5-P were quantified ( $n=3$, means \pm s.d.) and plotted in arbitrary units (a.u.). The results are representative of three independent experiments. Source data are provided as a Source Data file 
a

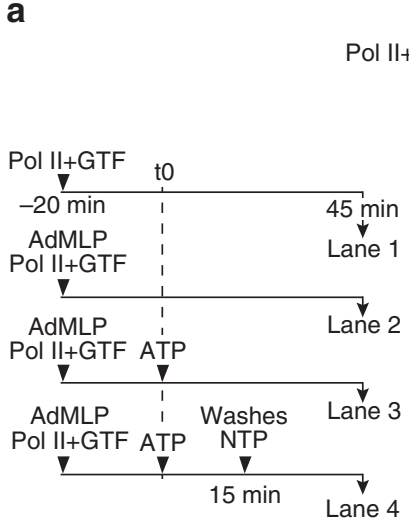

b
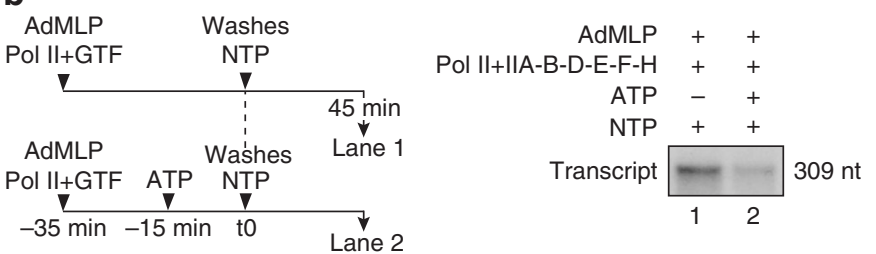

d

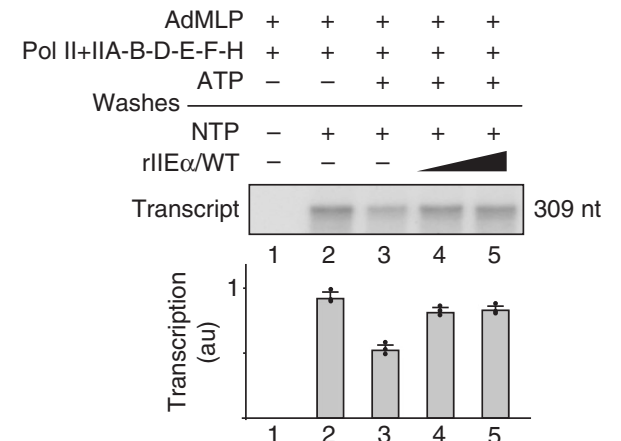

e AdMLP - + +

Pol II+IIA-B-D-E-F-H +++

WCE +++

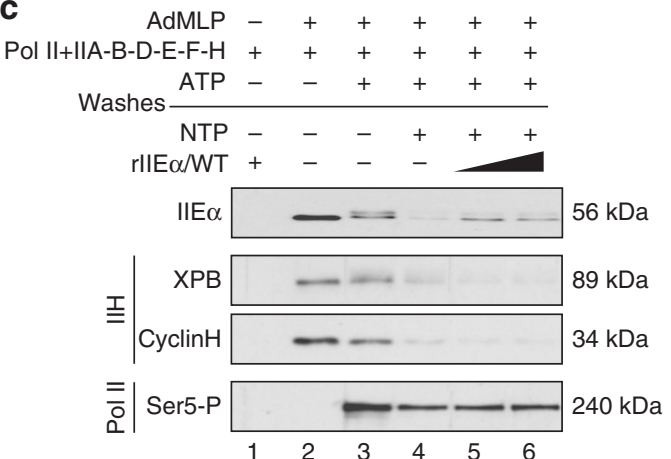

NTP - -+

W IIE $\beta \longrightarrow 34 \mathrm{kDa}$

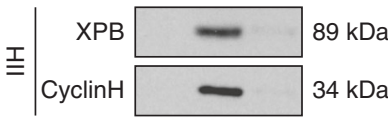

$\overline{\bar{O}} \mid$ Ser5-P $\square 240 \mathrm{kDa}$

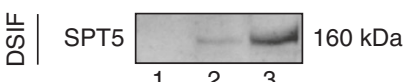

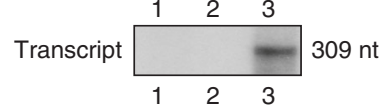

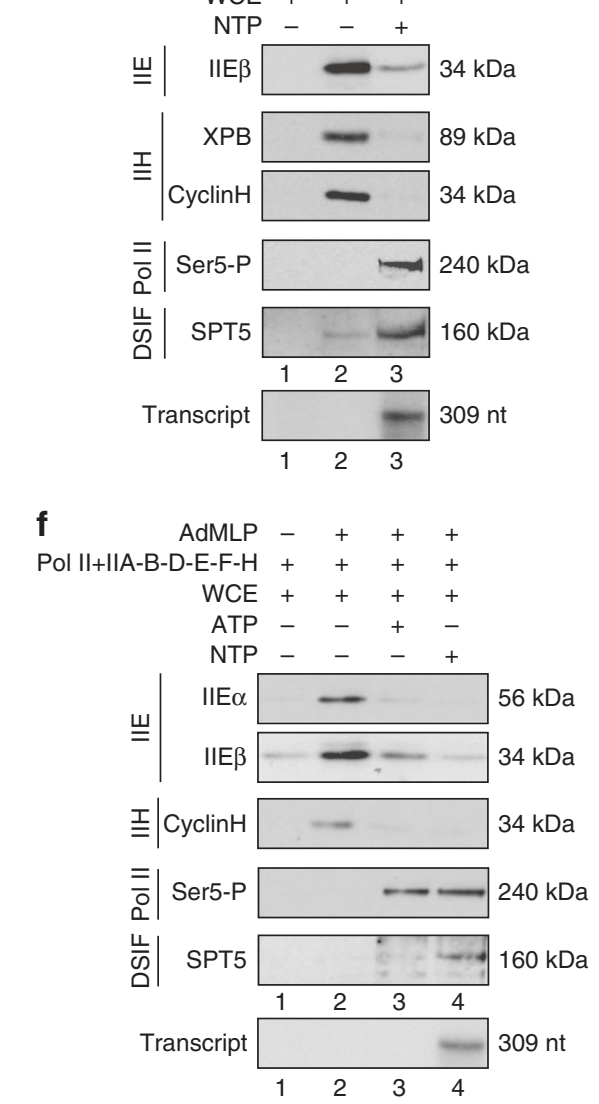

Fig. 5 The CAK is released during RNA synthesis. a Streptavidin magnetic beads were incubated (as depicted in the scheme, left part) with biotinylated AdMLP, Pol II, and the GTFs (TFIIA, TFIIB, TFIID (TBP), rlIE $\alpha \beta /$ WT, TFIIF, and TFIIH/WT), in presence of ATP (200 $\mu$ M). After 15 min of incubation with ATP, the beads were washed and NTPs $(200 \mu \mathrm{M}$ ) were added (lane 4). Immunoblot analysis (for TFIIE $\alpha$, TFIIE $\beta$, XPB, CyclinH, RPB1) and the transcription activity (309nt run-off transcript) were assessed after $45 \mathrm{~min}$ of incubation. The results are representative of three independent experiments. b Streptavidin magnetic beads with biotinylated AdMLP, Pol II, and the GTFs (TFIIA, TFIIB, TFIID (TBP), rIIE $\alpha \beta /$ WT, TFIIF, and TFIIH/WT) were pre incubated $15 \mathrm{~min}$ (as depicted in the scheme) in presence (lane 2) or absence (lane 1) of ATP $(200 \mu \mathrm{M})$. The beads were washed and NTPs (200 $\mu \mathrm{M}$ ) were added. Transcription activity (309nt run-off transcript) were assessed after $45 \mathrm{~min}$ of incubation. The results are representative of three independent experiments. c, d Streptavidin magnetic beads were pre incubated (when indicated, +) with biotinylated AdMLP, Pol II and the GTFs (TFIIA, TFIIB, TFIID (TBP), rlIE $\alpha \beta /$ WT, TFIIF, and TFIIH/WT) in presence of ATP $(200 \mu \mathrm{M})$. After washes, NTP $(200 \mu \mathrm{M})$ and increasing amounts of rlIE $\alpha / \mathrm{WT}$ were added. Immunoblot analysis (c) and transcription activity (d) were assessed after $45 \mathrm{~min}$ of incubation. The transcription activities were quantified $(n=3$, means \pm s.d.) and plotted in arbitrary units (a.u.). The results are representative of two independent experiments. e, $\mathbf{f}$ Streptavidin magnetic beads were incubated (when indicated, +) with biotinylated AdMLP, Pol II, TFIIA, TFIIB, TFIID (TBP), rIIE $\alpha \beta /$ WT, TFIIF, and TFIIH/WT, in presence of whole cell extracts (WCE), ATP $(200 \mu \mathrm{M})(\mathbf{f})$ and NTP $(200 \mu \mathrm{M})(\mathbf{e}, \mathbf{f})$. Immunoblot analysis (for TFIIE $\alpha$, TFIIE $\beta$, XPB, CyclinH, Ser5-P, and SPT5) and the transcription activity (309nt run-off transcript) were assessed after $45 \mathrm{~min}$ of incubation. The results are representative of three independent experiments. Source data are provided as a Source Data file 
a

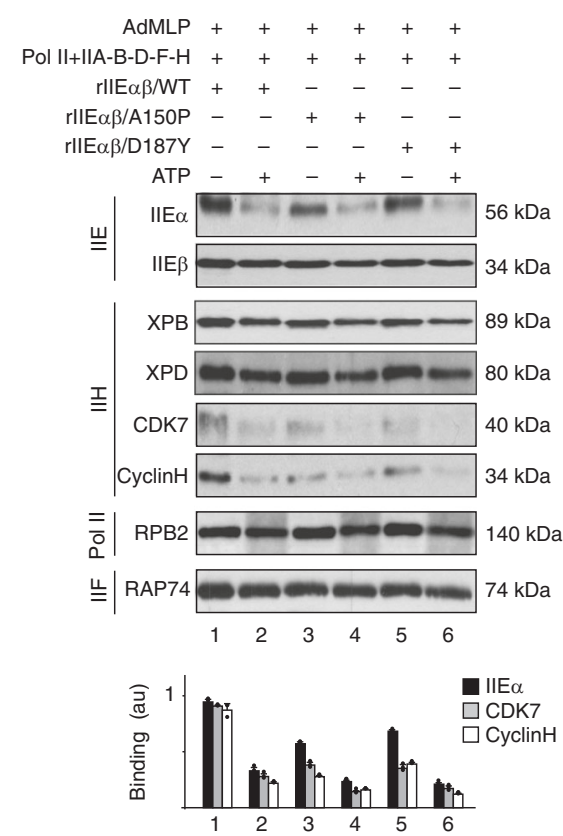

C

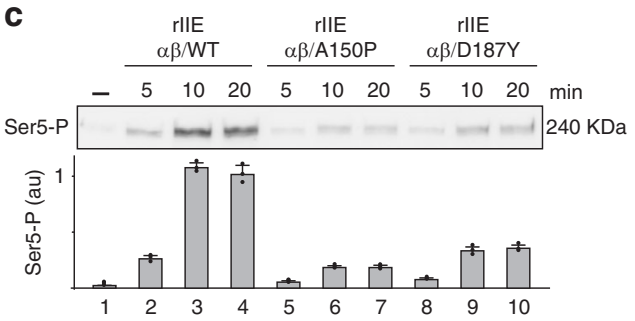

b
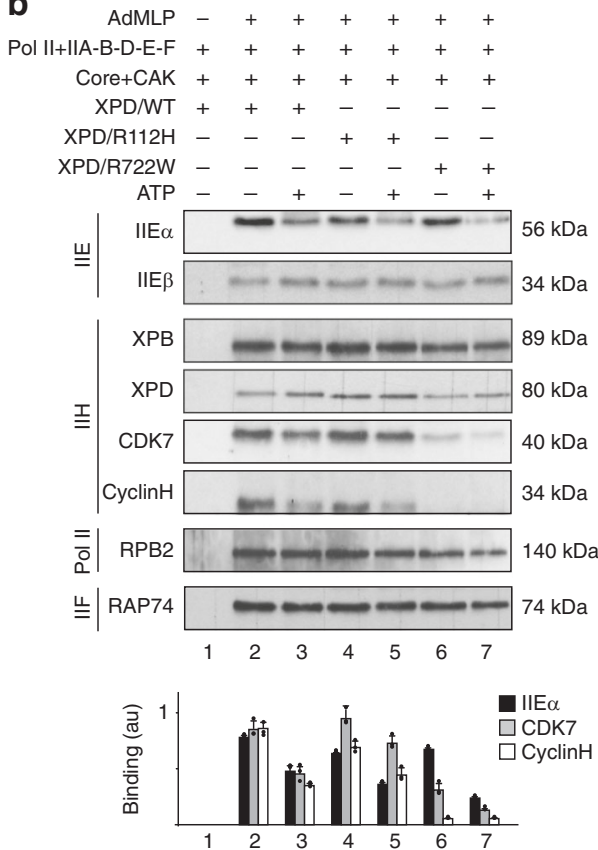

d

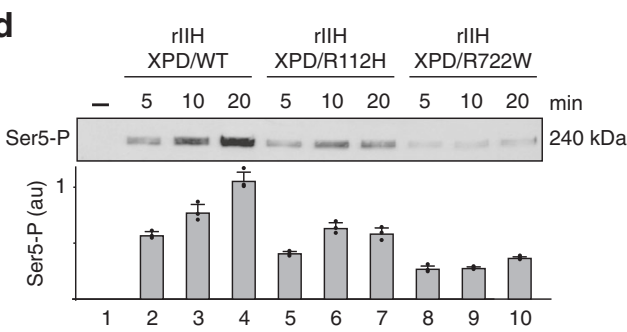

Fig. 6 TTD mutations alter CAK release and Pol II phosphorylation. a Biotinylated AdMLP bound to streptavidin magnetic beads was incubated with Pol II, TFIIA, TFIIB, TFIID (TBP), TFIIF, and TFIIH/WT, in presence (when indicated, +) of ATP $(200 \mu \mathrm{M}$ ) and either rIIE $\alpha \beta /$ WT, /A150P, or /D187Y. The immunoblot signals for TFIIE $\alpha, C D K 7$, and CyclinH were quantified and plotted in arbitrary units (a.u.). The results $(n=3$, means \pm s.d.) are representative of three independent experiments. b Streptavidin magnetic beads were incubated (when indicated, + ) with biotinylated AdMLP, Pol II, TFIIA, TFIIB, TFIID, rIIE $\alpha \beta /$ WT, TFIIF, the Core-TFIIH, and the CAK, in presence of ATP $(200 \mu \mathrm{M})$ and either XPD/WT, /R112H or /R722W. The immunoblot signals for TFIIE $\alpha$, CDK7, and $\mathrm{CyclinH}$ were quantified ( $n=3$, means \pm s.d.) and plotted in arbitrary units (a.u.). The results are representative of three independent experiments. c, d In vitro reconstituted transcription systems with rllEs (c) and rllHs (d) have been performed in presence of cold NTPs. Immunoblot analysis next has been done using specific antibody against Ser5-P of Pol II. Signals were quantified $(n=3$, means \pm s.d.) and plotted in arbitrary units (a.u.). The results are representative of three independent experiments. Source data are provided as a Source Data file

of rIIE $\beta / A 150 \mathrm{P}$ and /D187Y strongly prevented CAK-dependent Pol II phosphorylation (Fig. 6c, lanes 5-7 and 8-10, respectively). Diminished Pol II phosphorylation was also observed with mutated forms of $\mathrm{rIIH}$ (Fig. 6d), the defect being more pronounced for XPD/R722W than XPD/R112H (lanes 5-7 and 8-10, respectively).

Taken together, these results suggested that TFIIE $\beta$ and XPD mutations cause in common a perturbation of Pol II phosphorylation by the CAK.

\section{Discussion}

The present study aimed to dissect the interplay between TFIIE and TFIIH during transcription, from their integration within the PIC until their release before the upcoming recruitment of elongation factors. This work also revealed how TTD mutations within TFIIE and TFIIH disrupt such processes, resulting in a decrease in transcription.

Upon gene activation, the GTFs target the promoter in a sequential order, which is initiated by the recruitment of TFIID, TFIIA, TFIIB, and Pol II together with TFIIF ${ }^{1,2}$, the whole forming a structure ready to incorporate TFIIE and TFIIH
(Fig. 7). Using immobilized template and highly purified recombinant GTFs, we show that the TFIIEa subunit is recruited at the promoter via TFIIE $\beta$, the latter interacting with Pol II (Supplementary Fig. 6a) ${ }^{37}$. While the Core-TFIIH can target the PIC in absence of TFIIE, through partnerships with Pol II (Supplementary Fig. 6b), we demonstrate that TFIIEa promotes the incorporation of the CAK module of TFIIH within the PIC (Fig. 3c), which is in accordance with structural analyses ${ }^{35}$. The PIC formation assays questions about the retention, and loss, of transcriptional components while Pol II advances to transcribe and is retained on the DNA template (Fig. 5a, lane 4). In a reconstituted in vitro transcription assay, we cannot exclude Pol II retention at the end of the DNA template, a situation requiring additional factors for the release of the polymerase. Furthermore, formation of inactive complexes can occur, resulting from incorrectly folded proteins and/or incomplete PIC assembly. However, in our experimental conditions, almost all the Pol II was phosphorylated after ATP addition, which is illustrated by the complete conversion of RNA polymerase IIA to IIO (Fig. 5a, lane 3). This suggests that the majority of the polymerase bound to the DNA template may be integrated into complexes that allow 


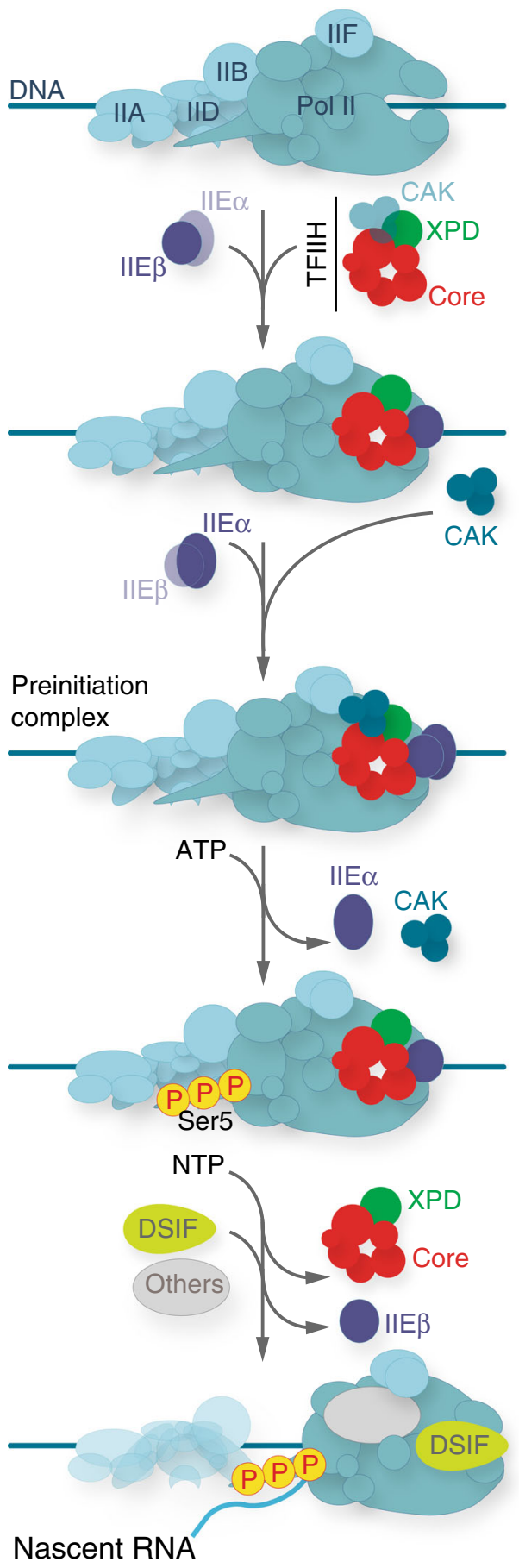

Fig. 7 Sequential association/dissociation of TFIIE and TFIIH. Once a stable TFIID-TFIIA-TFIIB-Pol II-TFIIF promoter complex is assembled, TFIIE and TFIIH can be recruited. The TFIIE $\beta$ subunit is required to anchor TFIIE $\alpha$. Altgough the Core-TFIIH sub-complex can incorporate the complex in absence of TFIIE, TFIIE $\alpha$, and TFIIE $\beta$ are both required to anchor the CAK within the PIC. In presence of ATP, Pol II phosphorylation is accompanied by the release of the CAK and TFIIE $\alpha$ from the promoter, a process that takes place before DNA opening. In presence of NTP, RNA synthesis is initiated, the Core-TFIIH and TFIIE $\beta$ are removed while DSIF and other elongation factors are recruited

it to become active. Interestingly, a selective (i.e. TFIIE and TFIIH) but not complete removal of GTFs was observed once Pol II is engaged in elongation (lane 4), as previously suggested ${ }^{47}$. Such dynamic recruitment/release of factors illustrates the complexity of the sequential events that are required to accomplish promoter clearance ${ }^{48}$.

Many questions remain unanswered about the fate of TFIIE and TFIIH during transcription initiation, their respective contributions being debated for a number of years ${ }^{6,33}$. Here we show that TFIIE allows the integration of the CAK within the PIC (Fig. 3c), leading to Pol II phosphorylation by CDK7 (Fig. 4a) ${ }^{9}$. Once Pol II is phosphorylated, TFIIEa and the CAK are released from the promoter (Fig. $4 \mathrm{a}-\mathrm{c}$ ). Interestingly, the CAK dissociation observed during transcription mirrors what happens during NER. Indeed, upon arrival of the NER factor XPA on the damaged DNA (already targeted by XPC and TFIIH), the CAK is released in an ATP-dependent manner from the Core-TFIIH ${ }^{49}$.

Our results show that Pol II phosphorylation, and release of TFIIE $\alpha$ and the CAK, do not require DNA opening (Fig. 4d-f), since these events are not impeded by TPL that inhibits XPB enzymatic activity ${ }^{40}$, or mutations in XPB that affect its promoter opening activity ${ }^{41-43}$.

The ATP-dependent release of TFIIEa and the CAK raises the question of the fate of the Core-TFIIH and TFIIE $\beta$ within the remaining complex. The Core-TFIIH is required for next transcriptional events; in particular, the translocase activity of XPB allows DNA pumping into the active site cleft of Pol $\mathrm{II}^{15,50}$. Interestingly, the Core-TFIIH is not longer associated with the promoter once RNA synthesis is undertaken (Fig. 5a). Concerning TFIIE $\beta$, this subunit interacts with DNA (Supplementary Fig. 7), which might prove useful during promoter opening $6,7,51$. TFIIE $\beta$ is also no longer maintained at the promoter (Figs. $1 \mathrm{~h}, \mathrm{i}, \mathrm{n}, 5 \mathrm{a}$ and Supplementary Fig. 1i, j); its release parallels the recruitment of elongation factors, such as DSIF (Figs. 1q, 5e, f), a heterodimer of SPT4 and SPT5 that mediates pausing by Pol II early after initiation $^{31}$. Remarkably, structural analyses showed that the stalk domain of Pol II binds TFIIE as well as DSIF ${ }^{52}$. The fact that DSIF and TFIIE bind overlapping sites in the clamp region of Pol II suggest that DSIF might substitute for TFIIE ${ }^{53-55}$, a situation strongly corroborated by our results (Fig. 5e). Interestingly, reductions in DSIF and increases in TFIIE near promoters have been described as a result of CDK7 inhibition ${ }^{56-58}$. Whether or not the eviction of TFIIE requires post translational modifications remains to be determined. In this regard, it should be pointed out that a doublet for TFIIEa was observed in presence of ATP (Figs. 4, 5 and 6). This might result from the phosphorylation of TFIIEa, likely by CDK7, as previously observed ${ }^{9,56}$. Although such apparent TFIIEa phosphorylation is of interest, substantial investigations should be undertaken to identify the phosphorylation(s) sites(s) and to determine the function(s) of such modification(s) during transcription.

The TFIIE $\beta / A 150 \mathrm{P}$ and /D187Y mutations have been recently associated with TTD syndrome ${ }^{25,26}$, which is so far mainly related to TFIIH mutations. The results presented here show that TTD-related mutations within TFIIE and TFIIH have in common (i) decreasing the cellular concentration of these complexes (Fig. 1a and Supplementary Fig. 1b) by weakening their stability (Fig. 3e, f), (ii) affecting the anchoring of the CAK within the PIC (Figs. 3a, g and 6a, b), (iii) hampering Pol II phosphorylation (Fig. 6c, d) and therefore (iv) disrupting transcription in an in vitro (Fig. 2c, d) and ex vivo context (Fig. 1b, c, j, Supplementary Fig. 1c, d). Consequently, mutations in either GTF2E2 (which affect the interaction of TFIIE $\beta$ with TFIIE $\alpha$, Fig. 3e) or $X P D$ (which disturb the TFIIH stability, Fig. 3f) share similar consequences, mainly the lack of recruitment of the CAK and of its activity during transcription. The fact that mutations in TFIIE do not alter NER (Supplementary Fig. 3a) support the idea that Trichothiodystrophy is mainly related to transcription deficiencies. Overall, TTD-related mutations in TFIIE and TFIIH lead to numerous similar impacts during transcription initiation, 
which might explain in part why mutations within two complexes can lead to the same transcriptional syndrome.

\section{Methods \\ Reagents and resources. The key reagents and resources (antibodies, chemical, cell lines, oligonucleotides, recombinant DNA, software, and materials) are given in} Supplementary Table 1 .

Cell culture. Cells were grown in DMEM/Ham-F10 (1:1) supplemented with $10 \%$ FCS and $40 \mu \mathrm{g} / \mathrm{ml}$ gentamicin. Treatments with t-RA $(10 \mu \mathrm{M})$ were performed in the presence of red phenol-free medium containing 5\% charcoal-treated FCS and $40 \mu \mathrm{g} / \mathrm{ml}$ gentamicin.

Generation of knock-in TFIIE $\beta$ /A150P cell line. Human osteosarcoma epithelial U2OS cells were co-transfected with the px2-Cas9WT(GFP)-Puro plasmid (overexpressing tagged GFP-Cas9 and functional guide RNA, gRNA) and a singlestranded oligodeoxynucleotide (ssODN) corresponding to an exon 5 region of GTF2E2 with the missense mutation c.448G $>$ C [p.Ala150Pro]. The GFP-positive cells were sorted $24 \mathrm{~h}$ post-transfection, and were diluted $48 \mathrm{~h}$ later as 0.5 cells/well in p96 plates. Cells were grown in DMEM ( $1 \mathrm{~g} / 1$ glucose) supplemented with $10 \%$ FCS and $40 \mu \mathrm{g} / \mathrm{ml}$ gentamicin. Clones were screened by PCR and enzymatic digestion followed by further sequencing to confirm the correct knock-in in the desired locus.

Retrotranscription and real-time qPCR. Total RNAs $(2 \mu \mathrm{g})$ were reverse transcribed with Moloney murine leukemia virus RT (Invitrogen) using random hexanucleotides. Real-time qPCR (denaturation at $95^{\circ} \mathrm{C}$ for $10 \mathrm{~s}$, annealing at $65^{\circ} \mathrm{C}$ for $15 \mathrm{~s}$, elongation at $72{ }^{\circ} \mathrm{C}$ for $15 \mathrm{~s}$ ) was done using the "FastStart DNA Master SYBR Green" kit and the LightCycler apparatus (Roche Diagnostics). The RAR $\beta 2$ mRNA expression represents the ratio between values obtained from treated and untreated cells normalized to 18S RNA. The primer sequences are given in Supplementary Table 1.

ChIP assays. Subconfluent cells were treated with t-RA $(10 \mu \mathrm{M})$ and ChIP experiments were next performed with Protein G-magnetic beads (Dynabeads, Invitrogen $)^{59}$. Non-specific controls were performed with samples incubated with magnetic beads in the absence of antibodies. Primers were designed to amplify regions located either at the proximal promoter or at exon 4 of RARB2 (see Supplementary Materials $)^{30}$. The primer sequences are given in Supplementary Table 1. PCR (denaturation at $95^{\circ} \mathrm{C}$ for $10 \mathrm{~s}$, annealing at $65^{\circ} \mathrm{C}$ for $15 \mathrm{~s}$, elongation at $72{ }^{\circ} \mathrm{C}$ for $15 \mathrm{~s}$ ) was done using the "FastStart DNA Master SYBR Green" kit and the LightCycler apparatus (Roche Diagnostics).

Construction and purification of rllEs and rllHs. Flag-TFIIEa as well as HisTFIIE//WT, /A150P and /D187Y were produced in E. coli. Baculoviruses overexpressing Flag-XPD/WT, /R112H, /R722W, as well as XPB, p62, p52, p44, p34, cdk7, cyclin H, MAT1, and p8 were previously generated ${ }^{60,61}$. To purify entire rIIHs or XPD alone, Sf21 insect cells were infected with the corresponding baculoviruses. The whole cell extracts (WCE) were then incubated with agarose beads bound to anti-M2-Flag antibody. Recombinant TFIIH and the different forms of XPD were eluted with an epitope peptide.

NER assays. The single lesion (Pt-GTG) plasmid was prepared as described elsewhere ${ }^{62}$. Dual incision assay was carried out in the presence of XPG, XPF/ERCC1, XPC/hHR23B, RPA, XPA, the different rIIHs and rIIEs (when indicated) ${ }^{61}$.

Transcription reactions. The AdMLP template was preincubated for $15 \mathrm{~min}$ at $25^{\circ} \mathrm{C}$ with Pol II, TFIIA, TBP, TFIIB, TFIIF, and the different rIIEs and rIIHs ${ }^{63}$ RNA synthesis was initiated by the addition of NTPs $(200 \mu \mathrm{M})$, including radiolabelled CTP $(0.15 \mu \mathrm{M})$. In order to study Pol II phosphorylation during transcription, classical run-off transcription assays were carried out in presence of cold NTPs; after SDS-PAGE, specific monoclonal antibody was used to reveal Ser5-P of Pol II

Abortive synthesis reactions. The AdMLP template was preincubated with the GTFs (Pol II, TBP, TFIIB, TFIIF, and the corresponding rIIEs and rIIHs) for 15 $\min$ at $25^{\circ} \mathrm{C}$. Phosphodiester bond synthesis was then initiated by the addition of priming dinucleotides CpA $(0.5 \mathrm{mM}), \mathrm{MgCl}_{2}(6.5 \mathrm{mM})$, dATP $(4 \mu \mathrm{M})$, and radiolabelled CTP $(1 \mu \mathrm{M})$. After $30 \mathrm{~min}$ of CpApC synthesis, the reactions were stopped in the presence of proteinase $\mathrm{K}(0.5 \mathrm{mg} / \mathrm{ml})$.

PIC formation assays. Biotinylated AdMLP bound to streptavidin magnetic beads was incubated $20 \mathrm{~min}$ at $25^{\circ} \mathrm{C}$ with Pol II, TFIIA, IIB, IIF, TBP, and the different rIIEs and rIIHs. After several washings at $50 \mathrm{mM} \mathrm{NaCl}$, the bound fractions were resolved by SDS-PAGE followed by immunoblottings. The abundance of each protein was assessed by immunoblot densitometry analysis (using ImageJ software). Each signal was quantified three times (mean \pm s.d.) and plotted in arbitrary units (a.u.)

Interaction assays. Depending on the coimmunoprecipitation assay, purified Pol II, rIIEs, and rIIHs were coincubated together with specific antibody (raised against either RPB1, XPD, p62, or Flag-Tag, as indicated) bound to protein G magnetic beads. After extensive washings (at either 150 or $500 \mathrm{mM} \mathrm{KCl}$ ), Western blots were performed using antibodies raised against the proteins of interest.

Reporting summary. Further information on research design is available in the Nature Research Reporting Summary linked to this article.

\section{Data availability}

All relevant data supporting the key findings of this study are available within the article and its Supplementary Information files or from the corresponding author upon reasonable request. The raw data underlying all reported averages in graphs are available upon request. The source data underlying Figs. 1-6 and Supplementary Figs. 1-7 are provided as a Source Data file. A Reporting Summary for this Article is available as a Supplementary Information file.

Received: 26 September 2018 Accepted: 18 April 2019 Published online: 07 May 2019

\section{References}

1. Maldonado, E., Ha, I., Cortes, P., Weis, L. \& Reinberg, D. Factors involved in specific transcription by mammalian RNA polymerase II: role of transcription factors IIA, IID, and IIB during formation of a transcription-competent complex. Mol. Cell. Biol. 10, 6335-6347 (1990).

2. Thomas, M. C. \& Chiang, C. M. The general transcription machinery and general cofactors. Crit. Rev. Biochem. Mol. Biol. 41, 105-178 (2006).

3. Peterson, M. G. et al. Structure and functional properties of human general transcription factor IIE. Nature 354, 369-373 (1991).

4. Watanabe, T. et al. The carboxy terminus of the small subunit of TFIIE regulates the transition from transcription initiation to elongation by RNA polymerase II. Mol. Cell. Biol. 23, 2914-2926 (2003).

5. Maxon, M. E., Goodrich, J. A. \& Tjian, R. Transcription factor IIE bind preferentially to RNA polymerase IIa and recruits TFIIH: a model for promoter clearance. Genes Dev. 8, 515-524 (1994).

6. Okamoto, T. et al. Analysis of the role of TFIIE in transcriptional regulation through structure-function studies of the TFIIEbeta subunit. J. Biol. Chem. 273, 19866-19876 (1998).

7. Kim, T. K., Ebright, R. H. \& Reinberg, D. Mechanism of ATP-dependent promoter melting by transcription factor IIH. Science 288, 1418-1422 (2000).

8. Forget, D., Langelier, M. F., Therien, C., Trinh, V. \& Coulombe, B. Photocross-linking of a purified preinitiation complex reveals central roles for the RNA polymerase II mobile clamp and TFIIE in initiation mechanisms. Mol. Cell. Biol. 24, 1122-1131 (2004).

9. Ohkuma, Y. \& Roeder, R. G. Regulation of TFIIH ATPase and kinase activities by TFIIE during active initiation complex formation. Nature 368, 160-163 (1994).

10. Sung, P. et al. Human xeroderma pigmentosum group D gene encodes a DNA helicase. Nature 365, 852-855 (1993).

11. Wang, Z. et al. Transcription factor b (TFIIH) is required during nucleotideexcision repair in yeast. Nature 368, 74-76 (1994).

12. Tirode, F., Busso, D., Coin, F. \& Egly, J. M. Reconstitution of the transcription factor TFIIH: assignment of functions for the three enzymatic subunits, XPB XPD, and cdk7. Mol. Cell 3, 87-95 (1999).

13. Holstege, F. C., van der Vliet, P. C. \& Timmers, H. T. Opening of an RNA polymerase II promoter occurs in two distinct steps and requires the basal transcription factors IIE and IIH. EMBO J. 15, 1666-1677 (1996).

14. Guzman, E. \& Lis, J. T. Transcription factor TFIIH is required for promoter melting in vivo. Mol. Cell. Biol. 19, 5652-5658 (1999).

15. Grunberg, S., Warfield, L. \& Hahn, S. Architecture of the RNA polymerase II preinitiation complex and mechanism of ATP-dependent promoter opening. Nat. Struct. Mol. Biol. 19, 788-796 (2012).

16. He, Y., Fang, J., Taatjes, D. J. \& Nogales, E. Structural visualization of key steps in human transcription initiation. Nature 495, 481-486 (2013).

17. Fishburn, J., Tomko, E., Galburt, E. \& Hahn, S. Double-stranded DNA translocase activity of transcription factor TFIIH and the mechanism of RNA polymerase II open complex formation. Proc. Natl Acad. Sci. USA 112, 3961-3966 (2015). 
18. Tomko, E. J., Fishburn, J., Hahn, S. \& Galburt, E. A. TFIIH generates a sixbase-pair open complex during RNAP II transcription initiation and start-site scanning. Nat. Struct. Mol. Biol. 24, 1139-1145 (2017).

19. Dvir, A., Conaway, R. C. \& Conaway, J. W. A role for TFIIH in controlling the activity of early RNA polymerase II elongation complexes. Proc. Natl.Acad. Sci. USA 94, 9006-9010 (1997).

20. Schwartz, B. E., Larochelle, S., Suter, B. \& Lis, J. T. Cdk7 is required for full activation of Drosophila heat shock genes and RNA polymerase II phosphorylation in vivo. Mol. Cell. Biol. 23, 6876-6886 (2003).

21. Wong, K. H., Jin, Y. \& Struhl, K. TFIIH phosphorylation of the Pol II CTD stimulates mediator dissociation from the preinitiation complex and promoter escape. Mol. Cell 54, 601-612 (2014).

22. Rochette-Egly, C., Adam, S., Rossignol, M., Egly, J. M. \& Chambon, P. Stimulation of RAR alpha activation function AF-1 through binding to the general transcription factor TFIIH and phosphorylation by CDK7. Cell 90, 97-107 (1997).

23. Compe, E. \& Egly, J. M. Nucleotide excision repair and transcriptional regulation: TFIIH and beyond. Annu. rev. Biochem. 85, 265-90 (2016)

24. Spaeth, J. M., Kim, N. H. \& Boyer, T. G. Mediator and human disease. Semin. Cell. Dev. Biol. 22, 776-787 (2011).

25. Kuschal, C. et al. GTF2E2 mutations destabilize the general transcription factor complex TFIIE in individuals with DNA repair-proficient trichothiodystrophy. Am. J. Hum. Genet. 98, 627-642 (2016).

26. Theil, A. F. et al. Trichothiodystrophy causative TFIIEbeta mutation affects transcription in highly differentiated tissue. Hum. Mol. Genet. 26, 4689-4698 (2017).

27. Faghri, S., Tamura, D., Kraemer, K. H. \& Digiovanna, J. J. Trichothiodystrophy: a systematic review of 112 published cases characterises a wide spectrum of clinical manifestations. J. Med. Genet. 45, 609-621 (2008).

28. Vermeulen, W. et al. Sublimiting concentration of TFIIH transcription/DNA repair factor causes TTD-A trichothiodystrophy disorder. Nat. Genet. 26, 307-313 (2000).

29. Botta, E. et al. Reduced level of the repair/transcription factor TFIIH in trichothiodystrophy. Hum. Mol. Genet. 11, 2919-2928 (2002).

30. Le May, N. et al. NER factors are recruited to active promoters and facilitate chromatin modification for transcription in the absence of exogenous genotoxic attack. Mol. Cell 38, 54-66 (2010).

31. Kwak, H. \& Lis, J. T. Control of transcriptional elongation. Annu. Rev. Genet. 47, 483-508 (2013).

32. Aboussekhra, A. et al. Mammalian DNA nucleotide excision repair reconstituted with purified protein components. Cell 80, 859-868 (1995).

33. Goodrich, J. A. \& Tjian, R. Transcription factors IIE and IIH and ATP hydrolysis direct promoter clearance by RNA polymerase II. Cell 77, 145-156 (1994).

34. Bradsher, J., Coin, F. \& Egly, J. M. Distinct roles for the helicases of TFIIH in transcript initiation and promoter escape. J. Biol. Chem. 275, 2532-2538 (2000).

35. Schilbach, S. et al. Structures of transcription pre-initiation complex with TFIIH and Mediator. Nature 551, 204-209 (2017).

36. Miwa, K. et al. Crystal structure of human general transcription factor TFIIE at atomic resolution. J. Mol. Biol. 428, 4258-4266 (2016).

37. He, Y. et al. Near-atomic resolution visualization of human transcription promoter opening. Nature 533, 359-365 (2016).

38. Lu, H., Zawel, L., Fisher, L., Egly, J. M. \& Reinberg, D. Human general transcription factor IIH phosphorylates the C-terminal domain of RNA polymerase II. Nature 358, 641-645 (1992)

39. Kwiatkowski, N. et al. Targeting transcription regulation in cancer with a covalent CDK7 inhibitor. Nature 511, 616-620 (2014)

40. Titov, D. V. et al. XPB, a subunit of TFIIH, is a target of the natural product triptolide. Nat. Chem. Biol. 7, 182-188 (2011).

41. Coin, F. et al. Phosphorylation of XPB helicase regulates TFIIH nucleotide excision repair activity. EMBO J. 23, 4835-4846 (2004).

42. Moreland, R. J. et al. A role for the TFIIH XPB DNA helicase in promoter escape by RNA polymerase II. J. Biol. Chem. 274, 22127-22130 (1999).

43. Oksenych, V., de Jesus, B. B., Zhovmer, A., Egly, J. M. \& Coin, F. Molecular insights into the recruitment of TFIIH to sites of DNA damage. EMBO J. 28, 2971-2980 (2009).

44. Dvir, A. et al. A role for ATP and TFIIH in activation of the RNA polymerase II preinitiation complex prior to transcription initiation. J. Biol. Chem. 271, 7245-7248 (1996).

45. Holstege, F. C., Fiedler, U. \& Timmers, H. T. Three transitions in the RNA polymerase II transcription complex during initiation. $E M B O \mathrm{~J} . \mathbf{1 6}$, 7468-7480 (1997).

46. Cabart, P. \& Luse, D. S. Inactivated RNA polymerase II open complexes can be reactivated with TFIIE. J. Biol. Chem. 287, 961-967 (2012).

47. Zawel, L., Kumar, K. P. \& Reinberg, D. Recycling of the general transcription factors during RNA polymerase II transcription. Genes Dev. 9, 1479-1490 (1995).
48. Luse, D. S. Promoter clearance by RNA polymerase II. Biochim. Biophys. Acta 1829, 63-68 (2013).

49. Coin, F. et al. Nucleotide excision repair driven by the dissociation of CAK from TFIIH. Mol. Cell 31, 9-20 (2008).

50. Murakami, K. et al. Architecture of an RNA polymerase II transcription preinitiation complex. Science 342, 1238724 (2013).

51. Okuda, M. et al. Structure of the central core domain of TFIIEbeta with a novel double-stranded DNA-binding surface. EMBO J. 19, 1346-1356 (2000).

52. Grohmann, D. et al. The initiation factor TFE and the elongation factor Spt $4 / 5$ compete for the RNAP clamp during transcription initiation and elongation. Mol. Cell 43, 263-274 (2011).

53. Klein, B. J. et al. RNA polymerase and transcription elongation factor Spt $4 / 5$ complex structure. Proc. Natl Acad. Sci. USA 108, 546-550 (2011).

54. Martinez-Rucobo, F. W., Sainsbury, S., Cheung, A. C. \& Cramer, P. Architecture of the RNA polymerase-Spt $4 / 5$ complex and basis of universal transcription processivity. EMBO J. 30, 1302-1310 (2011).

55. Plaschka, C. et al. Transcription initiation complex structures elucidate DNA opening. Nature 533, 353-358 (2016).

56. Larochelle, S. et al. Cyclin-dependent kinase control of the initiation-toelongation switch of RNA polymerase II. Nat. Struct. Mol. Biol. 19, 1108-1115 (2012).

57. Kelso, T. W. et al. Cyclin-dependent kinase 7 controls mRNA synthesis by affecting stability of preinitiation complexes, leading to altered gene expression, cell cycle progression, and survival of tumor cells. Mol. Cell. Biol. 34, 3675-3688 (2014).

58. Nilson, K. A. et al. THZ1 reveals roles for Cdk7 in Co-transcriptional capping and pausing. Mol. Cell 59, 576-587 (2015).

59. Singh, A., Compe, E., Le May, N. \& Egly, J. M. TFIIH subunit alterations causing xeroderma pigmentosum and trichothiodystrophy specifically disturb several steps during transcription. Am. J. Hum. Genet. 96, 194-207 (2015).

60. Coin, F., Oksenych, V. \& Egly, J. M. Distinct roles for the XPB/p52 and XPD/ p44 subcomplexes of TFIIH in damaged DNA opening during nucleotide excision repair. Mol. Cell 26, 245-256 (2007).

61. Dubaele, S. et al. Basal transcription defect discriminates between xeroderma pigmentosum and trichothiodystrophy in XPD patients. Mol. Cell 11, 1635-1646 (2003)

62. Araujo, S. J. et al. Nucleotide excision repair of DNA with recombinant human proteins: definition of the minimal set of factors, active forms of TFIIH, and modulation by CAK. Genes Dev. 14, 349-359 (2000).

63. Gerard, M. et al. Purification and interaction properties of the human RNA polymerase B(II) general transcription factor BTF2. J. Biol. Chem. 266 20940-20945 (1991).

\section{Acknowledgements}

We thank Miria Stefanini, Donata Orioli, Elena Botta, Christiane Kuschal, and Kenneth H. Kraemer for the different TTD cell lines; Charlotte Saint André, Philippe Catez, and Isabelle Kolb for their technical expertise; Bernardo Reina Saint Martin for setting up the CRISPR-Cas9 technique; Donata Orioli, Kenneth H. Kraemer, Arnaud Poterszman, and Kwang-Wok Choi for fruitful discussions; Thomas Sexton for carefully reading the manuscript. We also thank the IGBMC cell culture facility. This study was supported by l'Association pour la Recherche sur le Cancer (130607082), l'Institut National du Cancer (INCA-PLBIO17-043), la Ligue contre le cancer (Equipe labellisée 2019), the PICS-CNRS (no. 6824) and the Korean National Research Foundation for international collaboration (Global Research Laboratory program).

\section{Author contributions}

E.C., C.M.G, J.-M.E conceived and designed the experiments. E.C., C.M.G., and C.B. carried out the experiments. E.C., C.M.G., C.B., F.C., and J.-M.E. analyzed the data. E.C., F.C. and J.-M.E. contributed reagents, materials and analysis tools. E.C. and J.-M.E. wrote the paper.

\section{Additional information}

Supplementary Information accompanies this paper at https://doi.org/10.1038/s41467 019-10131-1.

Competing interests: The authors declare no competing interests.

Reprints and permission information is available online at http://npg.nature.com/ reprintsandpermissions/

Journal peer review information: Nature Communications thanks the anonymous reviewer(s) for their contribution to the peer review of this work. Peer reviewer reports are available.

Publisher's note: Springer Nature remains neutral with regard to jurisdictional claims in published maps and institutional affiliations. 
(c) (i) Open Access This article is licensed under a Creative Commons Attribution 4.0 International License, which permits use, sharing, adaptation, distribution and reproduction in any medium or format, as long as you give appropriate credit to the original author(s) and the source, provide a link to the Creative Commons license, and indicate if changes were made. The images or other third party material in this article are included in the article's Creative Commons license, unless indicated otherwise in a credit line to the material. If material is not included in the article's Creative Commons license and your intended use is not permitted by statutory regulation or exceeds the permitted use, you will need to obtain permission directly from the copyright holder. To view a copy of this license, visit http://creativecommons.org/ licenses/by/4.0/.

(C) The Author(s) 2019 